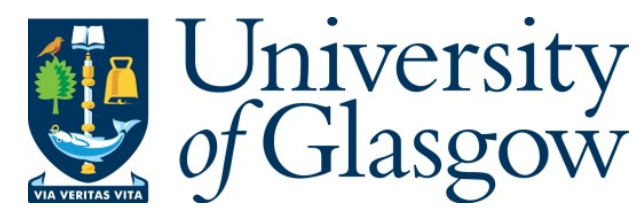

Ceriotti, M., and M clnnes, C. (2012) Natural and sail-displaced doublysymmetric Lagrange point orbits for polar coverage. Celestial M echanics and Dynamical A stronomy, 114 (1-2). pp. 151-180. ISSN 0923-2958

Copyright @ 2012 Springer V erlag

A copy can be downloaded for personal non-commercial research or study, without prior permission or charge

Content must not be changed in any way or reproduced in any format or medium without the formal permission of the copyright holder(s)

When referring to this work, full bibliographic details must be given

http://eprints.gla.ac.uk/65162/

Deposited on: 11 February 2014

Enlighten - Research publications by members of the U niversity of Glasgow http://eprints.gla.ac.uk 


\title{
Natural and sail-displaced doubly-symmetric Lagrange point orbits for polar coverage
}

\author{
Matteo Ceriotti (corresponding author) \\ Advanced Space Concepts Laboratory, Department of Mechanical \& Aerospace \\ Engineering \\ University of Strathclyde \\ 75 Montrose Street, James Weir Building \\ Glasgow G1 1XJ, United Kingdom \\ matteo.ceriotti@strath.ac.uk \\ Phone: +44 (0)141548 5726
}

Colin R. McInnes

Advanced Space Concepts Laboratory, Department of Mechanical \& Aerospace Engineering

University of Strathclyde

75 Montrose Street, James Weir Building

Glasgow G1 1XJ, United Kingdom

colin.mcinnes@strath.ac.uk

Phone: +44 (0)141 5482049

\section{Abstract}

This paper proposes the use of doubly-symmetric, eight-shaped orbits in the circular restricted three-body problem for continuous coverage of the high-latitude regions of the Earth. These orbits, for a range of amplitudes, spend a large fraction of their period above either pole of the Earth. It is shown that they complement Sun-synchronous polar and highly eccentric Molniya orbits, and present a possible alternative to low thrust pole-sitter orbits. Both natural and solar-sail displaced orbits are considered. Continuation methods are described and used to generate families of these orbits. Starting from ballistic orbits, other families are created either by increasing the sail lightness number, varying the period or changing the sail attitude. Some representative orbits are then chosen to demonstrate the visibility of high-latitude regions throughout the year. A stability analysis is also performed, revealing that the orbits are unstable: it is found that for particular orbits, a solar sail can reduce their instability. A preliminary design of a linear quadratic regulator is presented as a solution to stabilize the system by using the solar sail only. Finally, invariant manifolds are exploited to identify orbits that present the opportunity of a ballistic transfer directly from low Earth orbit. 
Keywords: Three-body problem, Eight-shaped orbits, Solar sailing, Polar observation, Periodic orbits

\section{Introduction}

Continuous polar coverage of the Earth's high-latitude regions could enable a range of applications that at the moment are only partially available: these include polar weather prediction, hurricane monitoring, and continuous telecommunication links with stations in the Antarctica (Lazzara et al. 2011). Currently, coverage of high-latitude regions of the Earth from space is performed by mainly two types of platforms. One consists of a spacecraft or a constellation in highly-inclined or polar, low or medium orbits. These orbits, such as Sunsynchronous orbits, allow the spacecraft to image only a narrow swath at each polar pass, relying on multiple passes for full coverage, therefore offering relatively low temporal resolution. The other option is to use highly elliptical orbits (HEOs). A particular type of HEO is the well-known Molniya orbit, whose period is typically one half of a sidereal day, and its inclination is $63.4^{\circ}$ or $116.6^{\circ}$. At either of these critical inclinations the argument of perigee no longer rotates due to Earth's oblateness, and the position of apogee remains unchanged (Wertz,Larson 1999). Although the apocentre is far enough to offer a hemispheric view of the Earth, the inclination is quite low for providing high-latitude continuous coverage.

Recent research (Anderson,Macdonald 2010) considered changing the critical inclination of the Molniya orbit to $90^{\circ}$, using a continuous solar electric propulsion (SEP) system for maintaining the orbit. However, the propellant mass fraction limits the mission duration and furthermore the spacecraft can only spend at most a few hours at the apocentre, at each passage.

The ideal point of view for viewing Earth's poles would be a stationary spacecraft above the pole itself. In this position, the footprint of the spacecraft will be constantly at the pole, in the same way as the footprint of a geostationary (GEO) spacecraft is constantly at some longitude on the equator.

This concept was originally proposed by Driver (1980), and named pole-sitter. However, while for GEO platforms the attraction of the Earth is balanced through the spacecraft's free-fall orbital motion, in a pole-sitter a relatively high, continuous acceleration must be provided to counterbalance Earth and solar 
gravitational attraction and maintain the spacecraft in such a location. This requires a low-thrust propulsion system continuously thrusting and it limits considerably the lifetime of the spacecraft.

Mainly to address these issues, different concepts have been proposed in the literature (Ceriotti et al. 2011). Some researchers proposed to use a solar sail to place a spacecraft (named Statite by Forward, 1991) on an artificial equilibrium point in the Sun-Earth system above $\mathrm{L}_{1}$, however the vertical displacement that can be achieved is relatively modest for realistic area-to-mass ratios (McInnes et al. 1994), and the coverage of the poles is not possible in certain seasons of the year. In order to maintain the latitude of the spacecraft as quasi-constant, a oneyear-periodic halo-type orbit around a displaced equilibrium point was designed by Waters,McInnes (2007). Other researchers instead investigate the use of hybrid solar sail propulsion and SEP to reduce the amount of propellant that is needed by a Statite (Baig,McInnes 2008) or an SEP pole-sitter (Ceriotti,McInnes 2011). In this paper, instead, we investigated a novel, alternative concept for highlatitude Earth coverage, which complements both the existing studies on polesitters and other stationary platforms in the Sun-Earth system, and existing Earthcentered missions on HEOs and polar orbits. The concept makes use of solar-saildisplaced doubly-symmetric orbits in the Sun-Earth system. Doubly-symmetric orbits (also called "type C" by Goudas, 1963) are periodic orbits that exist in the circular restricted three-body problem (CR3BP), and are symmetric with respect to two orthogonal planes. A particular kind of these orbits are eight-shaped (or figure-of-eight) orbits, which at small amplitudes appear in the vicinity of the collinear Lagrange points. Their name comes from their shape, which resembles a figure-eight when viewed in the $y$ - $z$ plane, at least for a certain range of amplitudes. The orbits were first discovered and sketched by Moulton (1920), however the computational capabilities at that time were not enough for a complete numerical integration. It was only more recently that, in a numerical survey of periodic orbits in the CR3BP, families of these orbits were precisely computed (Kazantzis 1979b, 1980).

It was firstly proposed to use these orbits for high-latitude observation for the first time by Folta et al. (2001). Recently, Archambeau et al. (2011) published a study on the same orbits in the Earth-moon system; in the same work, a detailed study of the manifolds of these orbits towards the moon was provided, with the idea of 
identifying possible trajectories that can be used for lunar observation. It is also found that manifolds of these orbits preserve their shape for a longer time than those of classic halo orbits.

On an eight-shaped orbit, a spacecraft would oscillate above and below the ecliptic plane, spending a long fraction of the orbit period above either pole, as will be shown. Therefore, for an interval of time, the spacecraft can offer similar services to those of a pole-sitter. In this paper, we will present the design of different families of natural and sail-displaced eight-shaped orbits, and will investigate the visibility conditions that they offer for a potential future platform for continuous polar coverage, constituted by one or a formation of spacecraft. We will also assess their natural instability, and show that the sail can contribute positively to mitigate it, for certain families of orbits and choice of lightness numbers.

Sail-displaced eight-shaped orbits were also studied by Farrés,Jorba (2010): in that paper the authors fixed the sail lightness number at 0.051689 (the one used for the Geostorm mission), a reasonable value for a near-term application, and families of orbits at $\mathrm{L}_{1}$ were designed by varying the out-of-plane angle of the solar sail with respect to the ecliptic plane.

In this paper, we consider the $\mathrm{L}_{2}$ case, because the sail displacement that is generated is towards the Earth, and therefore beneficial for Earth observation, as it will be explained. Furthermore, we additionally consider families of orbits with different lightness numbers, ranging from 0 (which represents the ballistic case) to 0.05 , and we will show that orbits interesting for polar observation can be found inside this range.

The present paper is organized as follows. Section 1 presents the methodologies to design families of natural and sail-displaced eight-shaped orbits; Section 2 studies the visibility conditions that some selected orbits offer; Section 3 describes the stability analysis of the orbit families, and a preliminary control method, based on a linear quadratic regulator $(\mathrm{LQR})$ is presented; finally, Section 3.1 studies the invariant manifolds of the orbits, to investigate the possibility of ballistic transfers from the Earth. 


\section{Eight-shaped orbit design}

We consider the CR3BP that describes the motion of a negligible mass (the spacecraft) under the gravitational attraction of two masses (the primaries, Sun and Earth in this case), with circular motion around their common centre-of-mass. We use a synodic reference frame centered at the center-of-mass, with the $\hat{\mathbf{x}}$ axis collinear with the two primaries, pointing towards the Earth, the $\hat{\mathbf{z}}$ axis is aligned with the angular velocity of the primaries $\boldsymbol{\omega}=\omega \hat{\mathbf{z}}$ and the $\hat{\mathbf{y}}$ axis completes the right-hand system (Fig. 1).

The dynamics of a spacecraft at position $\mathbf{r}$ is governed by the well known equation:

$$
\ddot{\mathbf{r}}+2 \boldsymbol{\omega} \times \dot{\mathbf{r}}-\nabla U=\mathbf{a}
$$

where $\mathbf{r}=[x, y, z]^{T}$ is the position vector and the effective potential, which takes into account gravitational attraction and centrifugal acceleration, is:

$$
U=\frac{1}{2}\left(x^{2}+y^{2}\right) \omega^{2}+\frac{\mu_{1}}{r_{1}}+\frac{\mu_{2}}{r_{2}}
$$

The two vectors $\mathbf{r}_{1}$ and $\mathbf{r}_{2}$ represent the position of the spacecraft with respect to the Sun and the Earth, respectively (see Fig. 1), and $\mu_{1}, \mu_{2}$ are their respective gravitational parameters.

Equation (1) will be used in its canonical non-dimensional form, which assumes $\omega=1, \mu=m_{2} /\left(m_{1}+m_{2}\right)$, and the unit of distance is the separation of the two primaries (1 Astronomical Unit). With these assumptions, the position along the $\hat{\mathbf{x}}$-axis of $m_{1}$ is $-\mu$, and the position of $m_{2}$ is $1-\mu$. Therefore we have $\mathbf{r}_{1}=[x+\mu, y, z]$ and $\mathbf{r}_{1}=[x-(1-\mu), y, z]$. For the Earth-Sun system, $\mu=3.0404 \cdot 10^{-6}$.

In the case of natural motion, the external acceleration term $\mathbf{a}$ is null. If instead the spacecraft is equipped with a propulsion system, then $\mathbf{a}$ is the acceleration provided by that system. In this paper, we will consider a solar sail. In the case of a flat, ideal sail, the non-dimensional acceleration can be expressed as (McInnes 1999):

$$
\mathbf{a}=\beta \frac{1-\mu}{r_{1}^{2}}\left(\hat{\mathbf{n}} \cdot \hat{\mathbf{r}}_{1}\right)^{2} \hat{\mathbf{n}}
$$


where $\hat{\mathbf{n}}$ is the unit vector normal to the sail surface and away from the Sun $\left(\hat{\mathbf{n}} \cdot \hat{\mathbf{r}}_{1}>0\right)$, and $\beta=\sigma^{*} A / m$ is the lightness number: proportional through $\sigma^{*} \cong 1.53 \cdot 10^{-3} \mathrm{~kg} / \mathrm{m}^{2}$ to the area-to-mass ratio of the sailcraft, it represents the ratio of solar pressure acceleration to the gravitational acceleration. It is usually taken as a technology parameter of the sailcraft, and values ranging from 0 (no sail) to 0.05 can be assumed for near- to mid-term technology (Dachwald et al. 2006).

Finally, it will become useful to rewrite Eq. (1) as a system of first-order equations, introducing the state vector $\mathbf{s}=\left[\begin{array}{llllll}x & y & z & v_{x} & v_{y} & v_{z}\end{array}\right]^{T}$ in the following form:

$$
\left\{\begin{array}{l}
\dot{x}=v_{x} \\
\dot{y}=v_{y} \\
\dot{z}=v_{z} \\
\dot{v}_{x}=2 v_{y}+x-\frac{1-\mu}{r_{1}^{3}}(x+\mu)-\frac{\mu}{r_{2}^{3}}(x-1+\mu)+\beta \frac{1-\mu}{r_{1}^{2}}\left(\hat{\mathbf{n}} \cdot \hat{\mathbf{r}}_{1}\right)^{2} n_{x} \\
\dot{v}_{y}=-2 v_{x}+y-\left[\frac{1-\mu}{r_{1}^{3}}+\frac{\mu}{r_{2}^{3}}\right] y+\beta \frac{1-\mu}{r_{1}^{2}}\left(\hat{\mathbf{n}} \cdot \hat{\mathbf{r}}_{1}\right)^{2} n_{y} \\
\dot{v}_{z}=-\left[\frac{1-\mu}{r_{1}^{3}}+\frac{\mu}{r_{2}^{3}}\right] z+\beta \frac{1-\mu}{r_{1}^{2}}\left(\hat{\mathbf{n}} \cdot \hat{\mathbf{r}}_{1}\right)^{2} n_{z}
\end{array}\right.
$$

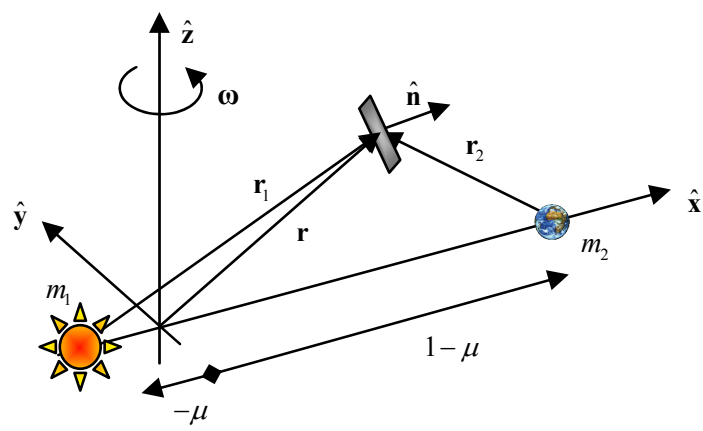

Fig. 1 Circular restricted three-body problem with solar sail

\subsection{Natural orbits}

Eight-shaped orbits are a particular type of the so called "type C" orbits by Kazantzis (1979a), which are symmetric both to the $x-y$ plane and the $x-z$ plane. It was also found by Kazantzis (1979b, 1980), following the work by Hénon (1974), that families of these types of orbits can be found through continuation, 
and the symmetry properties of eight-shaped orbits can be exploited for their design. In fact, each orbit crosses the $x-z$ plane four times in a period, and the $x$ axis twice. Fig. 2a represents an eight-shaped orbit after a quarter of its period. One of the intersections in which $z \neq 0$ (for example when $z>0$ ) can be taken as the origin of the orbit, and used for the predictor-corrector method that will be explained subsequently. By using the subscript " 0 " to identify this point, the initial state vector on the orbit has the form:

$$
\mathbf{s}_{0}=\left[\begin{array}{llllll}
x_{0} & 0 & z_{0} & 0 & v_{y 0} & 0
\end{array}\right]^{T}
$$

That is, due to the aforementioned symmetry, $y_{0}=v_{x 0}=v_{z 0}=0$. At the next intersection with the $x-z$ plane (subscript "1"), again due to symmetry considerations, the state vector is:

$$
\mathbf{s}_{1}=\left[\begin{array}{llllll}
x_{1} & 0 & 0 & 0 & v_{y 1} & v_{z 1}
\end{array}\right]^{T}
$$

This point corresponds to a quarter of the orbital period. Once $x_{0}, z_{0}, v_{y 0}$ are chosen such that $\mathbf{s}_{1}$ has the form of Eq. (5), then an integration of the initial conditions for a full period guarantees that the orbit is closed, periodic, and has the symmetry properties mentioned before. A predictor-corrector method can be implemented, to find the required initial conditions. Let us assume (at a generic step $i$ ) we have the initial state $\mathbf{s}_{0}^{(i)}$ of an eight-shaped orbit, and we would like to find another orbit, belonging to the same family, but with a slightly displaced initial position, to be determined:

$$
\mathbf{s}_{0}^{(i+1)}=\left[\begin{array}{c}
x_{0}^{(i)}+\delta x_{0} \\
0 \\
z_{0}^{(i)}+\delta z_{0} \\
0 \\
v_{y 0}^{(i)}+\delta v_{y 0} \\
0
\end{array}\right]
$$

The displacements $\delta x_{0}, \delta z_{0}, \delta v_{y 0}$ are unknown, and some constraint on $\delta x_{0}, \delta z_{0}$ shall be introduced to force the displacement of the initial point on the $x-z$ plane. For this, we introduce an angle $\alpha$ which identifies a direction in the $x-z$ plane with respect to the $+x$ axis, and then decomposes the displacement $\delta x_{0}, \delta z_{0}$ into a component $r_{1}$ along direction $\alpha$ and a component $r_{2}$ perpendicular to it (see Fig. 
2b). We can fix the value of $r_{1}$, being representative of the spacing between the solutions, and the direction $\alpha$ can be guessed from the previous iteration as:

$$
\alpha=\arctan \left[\left(z_{0}^{(i)}-z_{0}^{(i-1)}\right) /\left(x_{0}^{(i)}-x_{0}^{(i-1)}\right)\right]
$$

For the first iteration, the value of $\alpha$ has to be provided, starting from considerations on the dynamical system: for example, if a planar orbit is selected as a starting point, then a pure out-of-plane displacement is required for the first step, so $\alpha=\pi / 2$.

Through $\alpha, r_{1}, r_{2}$ we can re-write the displacements in position as:

$$
\begin{aligned}
& \delta x_{0}=r_{1} \cos (\alpha)+r_{2}^{(i+1)} \sin (\alpha) \\
& \delta z_{0}=z_{0}^{(i)}+r_{1} \sin (\alpha)-r_{2}^{(i+1)} \cos (\alpha)
\end{aligned}
$$

With this assumption, the unknown parameters are now $r_{2}^{(i+1)}, \delta v_{y 0}$. The natural first guess for these parameters is: $r_{2}^{(i+1)}=0$, which means that the direction of motion of the initial point does not change from the previous step; and $\delta v_{y 0}=0$, i.e. the initial velocity is the same as in the previous orbit (see again Fig. 2b). These assumptions are reasonable if the distance $r_{1}$ is small enough. At this point, we have a full guess for the initial state on the new orbit. This completes the predictor step of the method.

To determine the actual values of $r_{2}^{(i+1)}, v_{y 0}^{(i+1)}$, the corrector step is employed. This is an iterative procedure on its own. We numerically integrate the equations of motion (3), with no sail, starting from the initial condition, until the first intersection of the $x-z$ plane. The idea is to adjust these values in order to satisfy periodicity and symmetry conditions on the new orbit:

$$
\begin{aligned}
& y_{1}\left(x_{0}, z_{0}, v_{y 0}\right)=0 \\
& z_{1}\left(x_{0}, z_{0}, v_{y 0}\right)=0 \\
& v_{x 1}\left(x_{0}, z_{0}, v_{y 0}\right)=0
\end{aligned}
$$

In these equations, it is written explicitly that these values depend on the variables of the initial state $\mathbf{s}_{0}^{(i+1)}$. The condition (8) is automatically satisfied due to the integration stopping condition. The other two conditions can be used to correct 
$\delta r_{2}, \delta v_{y 0}$. In general, the state at the end of the integration will not match exactly the conditions, thus having:

$$
\begin{gathered}
z_{1}\left(\delta r_{2}, \delta v_{y 0}\right)=\delta z_{1} \\
v_{x 1}\left(\delta r_{2}, \delta v_{y 0}\right)=\delta v_{x 1}
\end{gathered}
$$

The correct values of $\delta r_{2}, \delta v_{y 0}$ can be found, for example, linearizing (Zagouras,Markellos 1977) the periodicity conditions (11)-(12), evaluating the derivatives through numerical integration, solving the corresponding system, finding updated values of $\delta r_{2}, \delta v_{y 0}$ and then repeating until $\delta z_{1} \cong \delta v_{x 1} \cong 0$ within a tolerance of $10^{-10}$ on both the function value and the step size. The numerical integration of the equations of motion is made through an explicit Runge-Kutta $(4,5)$ formula, implemented in MATLAB ode45 function, using $10^{-10}$ as relative and absolute tolerance.

After convergence of the corrector step, the initial state of the new orbit $\mathbf{s}_{0}^{(i+1)}$ can be determined thanks to Eq. (6) and (7), and the following iteration can be started with a new predictor step.

The whole predictor-corrector process is initiated using the initial state $\mathbf{s}_{0}^{(0)}$ on a known orbit, and an initial search direction $\alpha$. For example, it was shown by Kazantzis $(1979 b, 1980)$ that type-C orbits bifurcate out-of-plane from particular planar "vertical critical" orbits, and they have twice the multiplicity (and hence twice the period) of the planar orbits from which they bifurcate, when they are infinitesimally close to them. In the case of the eight-shaped orbits, the verticalcritical orbit is one of high amplitude that goes around the Sun, and this can be used to initiate the predictor-corrector procedure. The planar vertical critical orbits can be computed as described by Hénon (1974).

It is also known from the literature that the other end of the family does not collapse into a planar orbit, but rather into a vertical libration orbit at $\mathrm{L}_{1}$ or $\mathrm{L}_{2}$. Libration orbits of infinitesimal amplitude can be determined in the vicinity of the libration points through a dynamical system approach (Howell 2001) in a linear approximation, or small-amplitude orbits can be computed through a third-order Lindstedt-Poincaré method (Archambeau et al. 2011). Either of these methods can provide a good approximation for starting the first step of the predictor-corrector method. 
Note that the convergence of the corrector step relies on the selection of an appropriate value for $r_{1}$ : within the same orbit family, large values of $r_{1}$ may suffice for convergence in certain regions of the phase space, while it might be required to reduce $r_{1}$ in the vicinity of singularities or bifurcations.

The predictor-corrector procedure described here is different to the one described, for example, by Kazantzis (1980). In that work, the parameter $z_{0}$ is used for continuation: an arbitrary displacement $h$ in this component is set at each continuation step. However, as stated in the paper, along the family it might happen that $z_{0}$ reaches a maximum or a minimum: in that circumstance, it is not possible to find any other orbit by increasing (or decreasing respectively) this parameter. The author therefore suggests that when this happens, it is necessary to switch to another component, $x_{0}$ in this case. However this requires the detection of the singularity, which is not easily automated. Instead, with the proposed continuation, the direction of displacement of the initial point follows naturally the path, without any need of switching. This procedure is similar to the pseudoarclength continuation method proposed by Dichmann et al. (2002).

The reason why it was chosen to decompose the displacement into two perpendicular components, rather than for example fixing $r_{1}$ and allowing $\alpha$ to change, is avoid to converge on solutions whose initial point is in a direction perpendicular to the one of the previous step (for example at bifurcations).

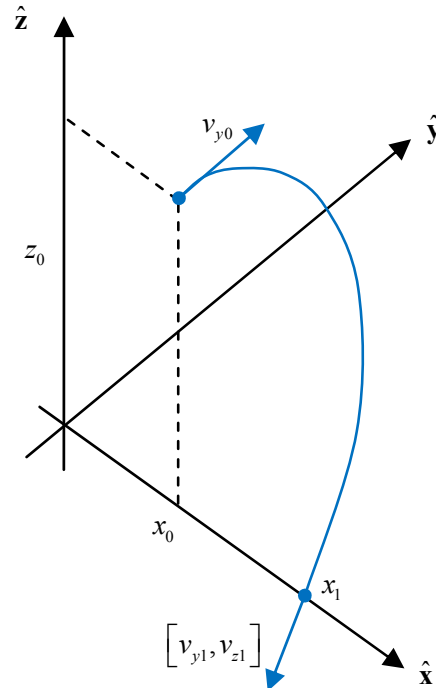

a)

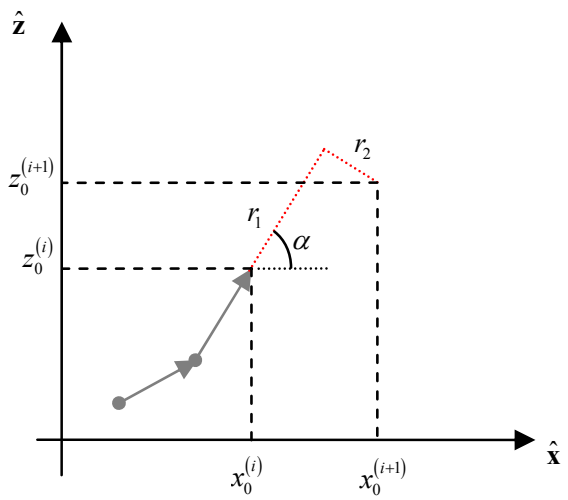

b)

Fig. 2 (a) A quarter period of an eight-shaped orbit. (b) Continuation method for type-C orbit 
By applying this method, the whole family of eight-shaped orbits at $L_{2}$ was designed. Some of these orbits are plotted in Fig. 3, in terms of their projections on the Cartesian orthogonal planes of the synodic frame. In the same figure the cone described by the polar axis of the Earth during one full year is plotted. The polar axis of the Earth maintains its orientation in an inertial frame (nutation and precession of equinoxes are not considered, as they are on a very long time scale), and therefore in the synodic reference frame it appears that the same axis has an apparent precession. A pole-sitter spacecraft would follow a trajectory on this cone, in order to continuously track the polar axis at each instant of time. In the case of the eight-shaper orbits, this is not possible; however the objective is to follow the cone as much as possible, for a long fraction of the orbit period, such that the visibility of the high-latitude regions is maximized and similar to that achieved by a pole-sitter.

It can be seen that the family collapses on $\mathrm{L}_{2}$, with purely vertical orbits, as described by Howell (2001). As the amplitude increases, the eight shape becomes well defined. Despite the family continuing with increasing $z$ amplitude, we limit the amplitude as the distance from the Earth becomes too high for useful applications. The complete family is plotted by Kazantzis (1979b).

Fig. 4 shows the period of the orbits in the family, as function of the $z$ amplitude. Again, as predicted in a dynamical system analysis (Howell 2001), the period of infinitesimal orbits approximates $\pi$. The period then increases with amplitude, however it does not reach one year (or $2 \pi$ ) in the interval of amplitudes considered. 


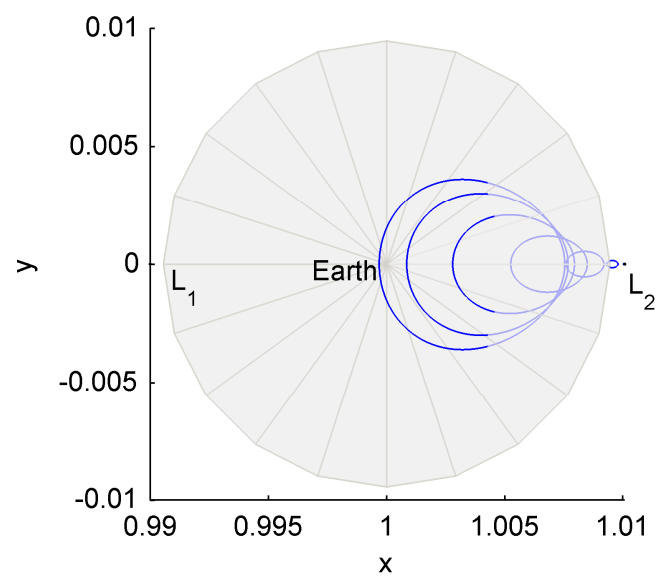

a)

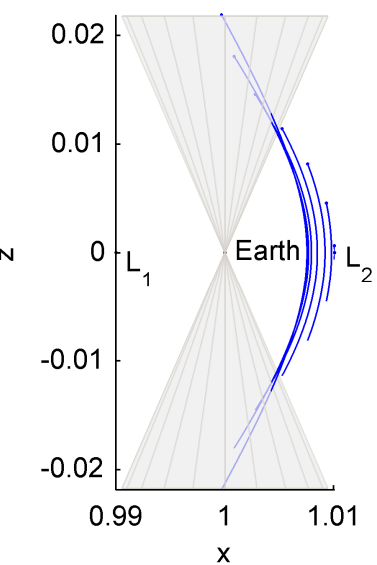

b)

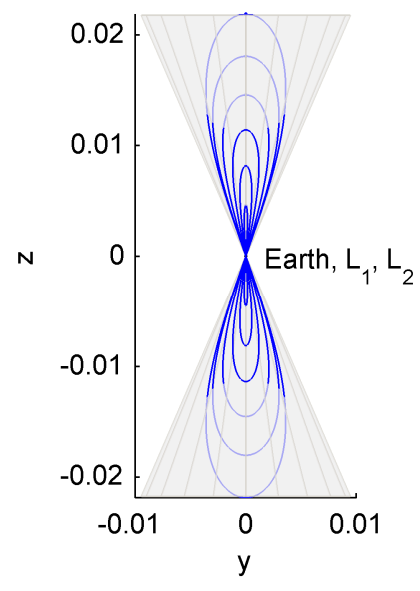

Fig. 3 Projections of some orbits of the family of natural eight-shaped orbits at $L_{2}$, with different $z$ amplitudes. (a) $x-y$ plane; (b) $x-z$ plane; (c) $y-z$ plane. Dots are the initial points

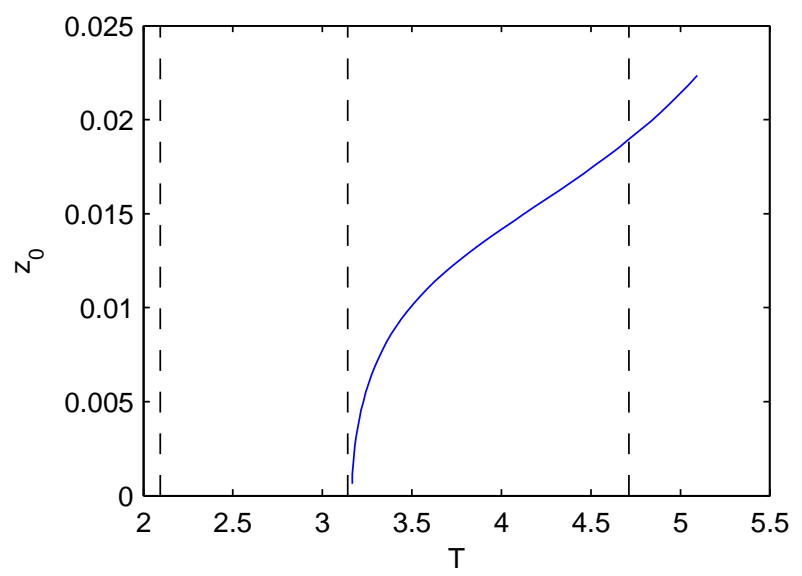

Fig. 4 Period of natural eight-shaped orbits, as function of the $z$ amplitude

The plots in Fig. 5 show the velocity along one of the orbits described, that with greatest amplitude. In particular it can be noted that the spacecraft motion is relatively slow around the northern and southern points of the orbit, while it is fast while crossing the ecliptic plane. This trend, combined with the shape of the orbit itself, justifies the choice of investigating this kind of orbits for polar observation: 
the spacecraft spends a consistent fraction of the orbital period above either of the poles of the Earth.

a)
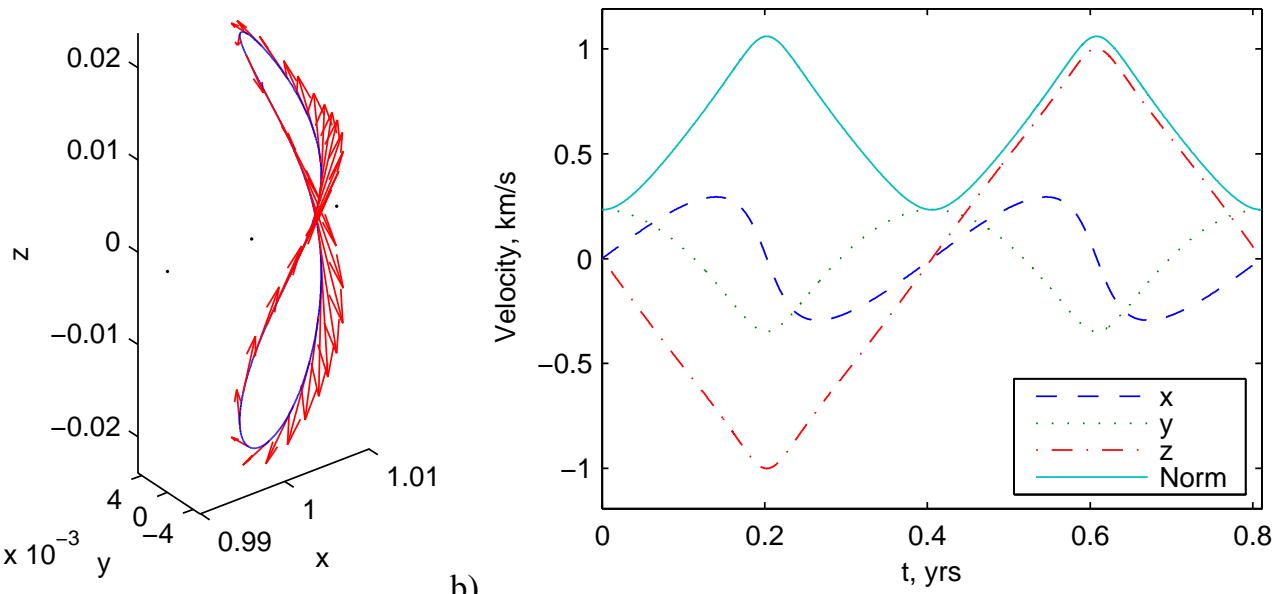

b)

elocity vector along a natural eight-shaped orbit. (a) Velocity vector plotted along the orbit; (b) velocity components and magnitude for an orbital period. $t=0$ corresponds to the top point on the orbit

Although the orbits presented here are at Lagrange point $\mathrm{L}_{2}$, the same procedure can be used to find orbits at $\mathrm{L}_{1}$. For the range of amplitudes considered here, it is found that these orbits are almost symmetrical with respect to the Earth to those at $\mathrm{L}_{2}$. For this reason, they are not shown here. Nevertheless, we investigate the $\mathrm{L}_{2}$ case rather than the $\mathrm{L}_{1}$ case because it is much more interesting when solar-saildisplaced orbits are considered, as shown in the following section.

\subsection{Sail-displaced orbits}

It is well known that the classical Lagrange points can be displaced if an external acceleration term is introduced into the equations of motion, i.e. a in Eq. (1). This is easy to show for example considering a constant and uniform acceleration along the $x$ axis; in this case, the collinear Lagrange points shift along the $x$ axis. Fig. 6 shows the position of the (artificial) Lagrange points $\mathrm{L}_{1}$ and $\mathrm{L}_{2}$ with respect to the Earth, for a range of accelerations. 


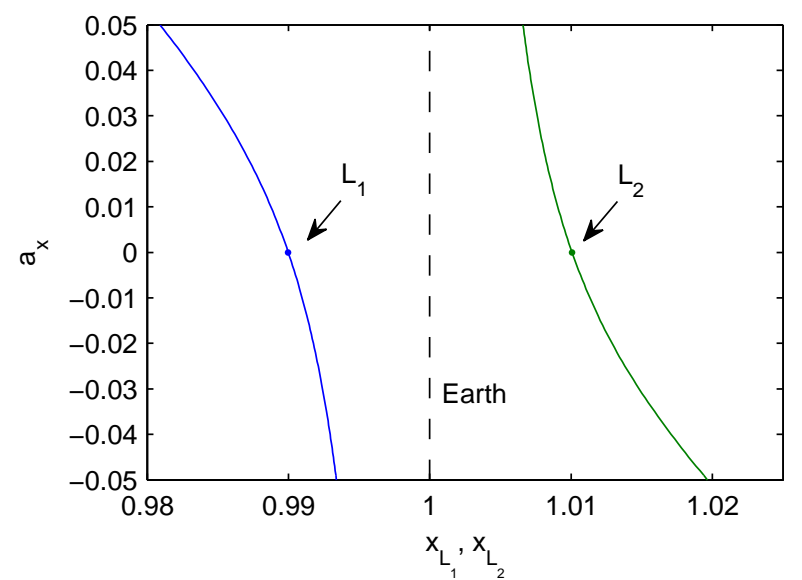

Fig. 6 Displacement of $L_{1}$ and $L_{2}$ points under a uniform and constant acceleration in the $x$ direction

Within the CR3BP including uniform acceleration, it is also possible to generate families of libration point orbits, such as halo orbits, around the displaced equilibria, as shown by Baig,McInnes (2009). Baoyin,McInnes (2005), and later Waters,McInnes (2007) demonstrated that those orbits exist also when a solar sail is considered.

It was mentioned in the Introduction that the analysis is performed for the $\mathrm{L}_{2}$ case. This is justified by the fact that, since the solar radiation pressure displaces orbits near the collinear equilibria towards the Sun, the orbits are displaced away from the Earth at $\mathrm{L}_{1}$, and towards the Earth at $\mathrm{L}_{2}$. The effect is clearly visible considering the Lagrange point itself, as in Fig. 6, but also libration point orbits (Baig,McInnes 2009).

In the first instance, we consider the sail with a fixed orientation in the synodic reference frame, and in particular with $\hat{\mathbf{n}}=\left[\begin{array}{lll}1 & 0 & 0\end{array}\right]^{T}$. This assumption is justified in two ways. The first is that this sail orientation maximizes the solar radiation pressure force and therefore the collinear displacement, for smallamplitude orbits around Lagrange points. In fact, the sail force is almost uniform and therefore the effect is similar to that of a uniform acceleration along the $x$ axis. The second reason is that the symmetry properties of the orbit are conserved. Note that the sail orientation is only fixed in the synodic frame, however the sail rotates with one full revolution every year in an inertial reference frame. One way to design solar-sail-displaced orbits is by repeating the same continuation procedure, but considering the full equations of motion, including the sail acceleration in Eq. (2). Instead, we start from the family of natural orbits 
found in the previous section, which can be seen as a special case of solar sail orbits in which $\beta=0$. The parameter $\beta$ can be used for continuation, therefore increasing the lightness number.

Since all symmetries of the eight-shaped orbits are preserved for the particular selected attitude of the solar sail, then the initial state and the state at $T / 4$ is still in the form of Eqs. (4)-(5). The difference with respect to the previous case is that, instead of using a pre-assigned displacement for the initial point (which was $r_{1}$ ), we perform the continuation on the parameter $\beta$. Since $r_{1}$ cannot be specified $a$ priori, then the three non-null initial states in $\mathbf{s}_{0}$ (Eq. (4)) are to be determined. However, there are only two conditions that are to be satisfied (namely Eqs. (9)(10)), since as explained earlier. the integration is stopped the first time that $y=0$. This condition can be reintroduced if the period of the orbit $T$ along the family is fixed. In the corrector step, the integration is stopped after $T / 4$, and that state corresponds now to $\mathbf{s}_{1}$. Therefore, all three conditions (8)-(10) are not trivial, and they can be used to find $x_{0}, z_{0}, v_{y 0}$. The $3 \times 3$ system can be linearized and solved iteratively in the same way as described earlier.

The next predictor step starts after incrementing $\beta$ a sufficiently small amount, and the procedure is repeated, using the previous initial state as a first guess. The increment on $\beta$ has the same role as $r_{1}$ in the previous case: therefore, we ensure that it is small enough to guarantee the convergence of each corrector step. This predictor-corrector step generates orbits with different lightness numbers, but constant period, along the family.

\subsection{Orbit families}

By using the two continuation procedures described here, and switching from one to the other when suitable values of the parameters (period or lightness number) are met, it is possible to design a number of families with constant lightness number (but varying period) and constant period (but varying lightness number). For the families with fixed period, some specific values of $T$ need to be selected. It is interesting to consider orbits that repeat with the same visibility conditions each year. One year is in fact the period of the apparent precession of the polar axis of the Earth in the synodic frame. We neglect here the daily rotation of the 
Earth, because we are not interested in following one specific point on the Earth's surface, but rather an area around the pole. This will become clear when taking into account the visibility angles in Section 2. A spacecraft, or a constellation, shall then have a suitable period such that the same relative configuration between the polar axis and the spacecraft is repeated every year.

In general, a spacecraft on a periodic orbit repeats the same visibility conditions if its orbit has the period of $2 \pi$ (or 1 year), or any submultiple $m$. If a constellation of $n$ spacecraft are used on the same orbit, and they are equally spaced in time, then the period of the orbit $T$ for repeating the same visibility conditions is:

$$
T=2 \pi \frac{n}{m}, \quad m \in 1,2,3, \ldots
$$

Note however that depending on $m$ and $n$, each spacecraft is not necessarily in the same position every year.

In this paper, three values of the period are chosen: $T=2 \pi / 3, \pi, 3 \pi / 2$ (corresponding to 4, 6 and 9 months respectively). In the first and second case, one spacecraft is sufficient to repeat the same observation every year ( $n=1, m=3,2)$, although more spacecraft can be used for additional coverage. In the last case, instead, a minimum of three spacecraft are necessary $(n=3, m=4)$. Periods shorter than 4 months do not seem to be suitable for offering a platform for quasi-static polar observation, while longer periods appear in orbits with very high $z$ amplitude, and therefore they are not suitable for Earth observation. The following figures aim at illustrating the different orbit families that were found. The first set of plots, grouped in Fig. 7, refer to the families that were found by moving the starting point in the $x-z$ plane, and letting the orbital period change, while keeping the sail orientation and lightness number fixed. The plot in Fig. 7a represents the $x-z$ synodic plane, and contains the starting points $\left(x_{0}, z_{0}\right)$ of the orbits of each family, parameterized by lightness number. Note that the coordinates $\left(x_{0}, z_{0}\right)$ are not sufficient to fully define the orbit, but the velocity $v_{y 0}$ is also needed to describe the initial state $\mathbf{s}_{0}$ as in Eq. (4). Nonetheless, the coordinates are helpful to immediately visualize the position of the apex of the orbit, around which the spacecraft can observe the polar regions. The first family in the plot is $\beta=0$ : this is the natural case; no sail is necessary for generating these orbits. This coincides with the family presented in Fig. 3. The dashed lines 
represent the polar axis of the Earth at the northern summer and winter solstices, respectively. Gray lines with no markers define the starting points of other families, that will be described later in this section, and they are plotted here as a reference.

Fig. $7 \mathrm{~b}$ is a three-dimensional representation of some orbits for each family, in the Sun-Earth synodic reference frame. The shaded conical surface is that described by the polar axis of the Earth in a one-year period, and it is plotted as the ideal condition for an orbit that could track the cone during the whole year, as for a pole-sitter spacecraft.

The period of each orbit is plotted in Fig. 7c, as a function of the $z$ amplitude, $z_{0}$. Note however that not all the families are monotonic in $z_{0}$, therefore in the same family there could be two orbits with the same $z_{0}$ but different period (they also have different $x_{0}$ and $v_{y 0}$ ). As a reference, vertical black dashed lines are drawn at the significant values of the period mentioned before. 


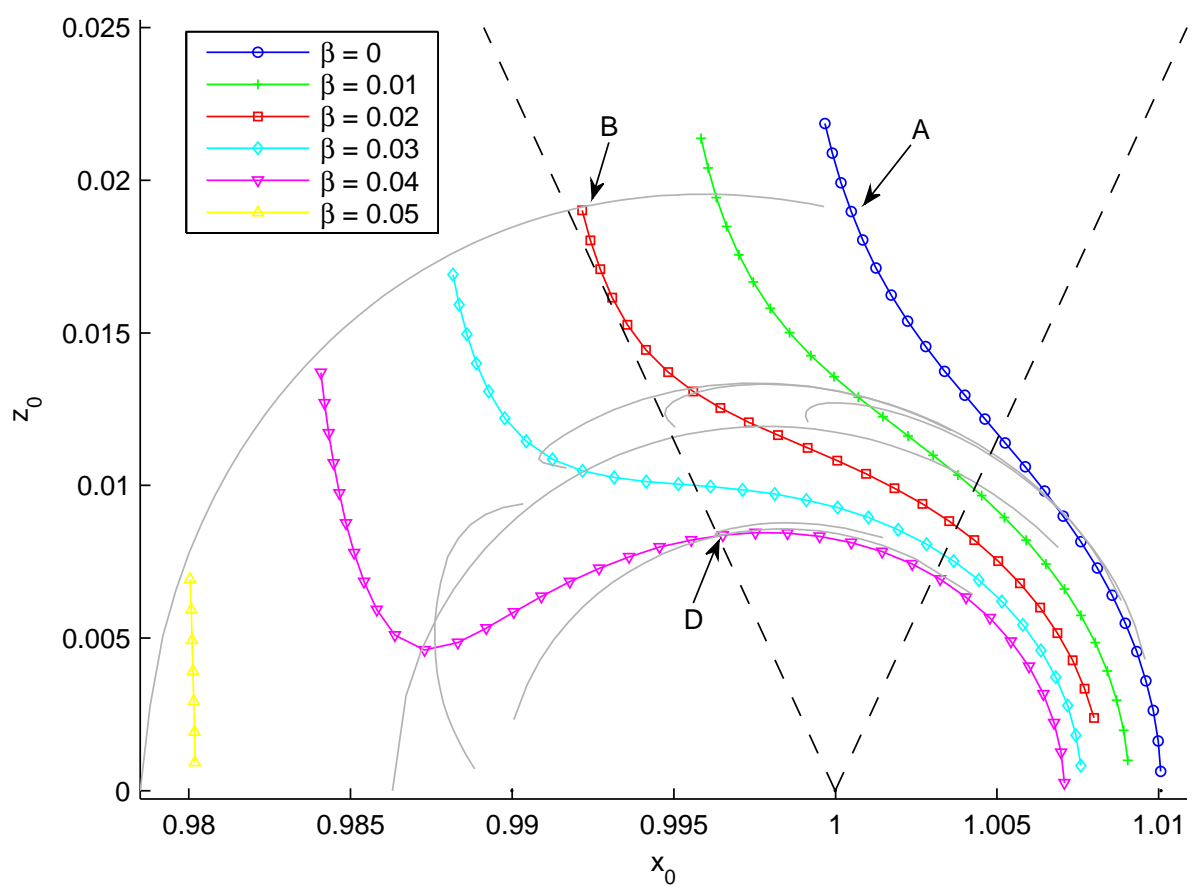

a)

b)
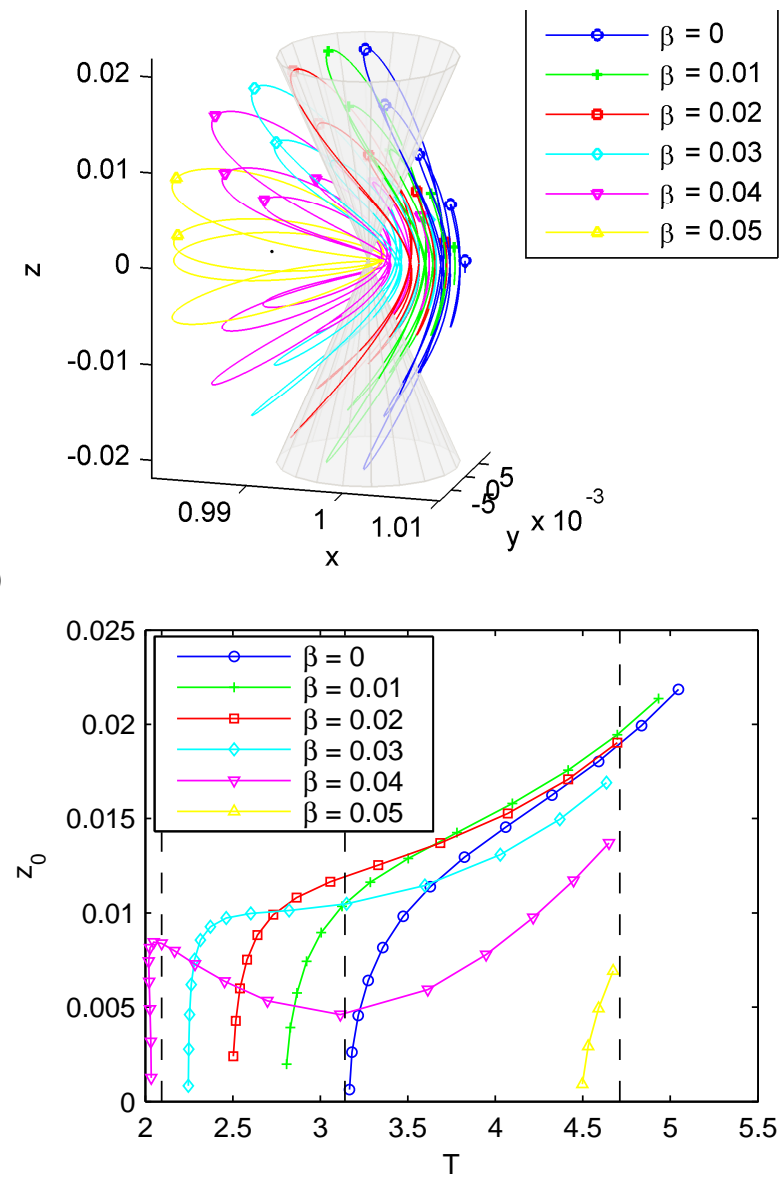

Fig. 7 Families of orbits with constant sail lightness number $\beta$. (a) Initial points in the $x-z$ plane. Gray lines represent other families of orbits presented in the paper, and are plotted as a reference; (b) Some of the orbits. (c) Period versus $z$ amplitude

The following Fig. 8 describes families of orbits with constant period and sail attitude, in which each orbit within a family differ for the sail lightness number. 
Fig. $8 \mathrm{a}$ is a plot of the starting points: families $T=3 \pi / 2$ and $T=\pi$ have their origin on the natural orbit family $\beta=0$. The latter in particular starts exactly at $\mathrm{L}_{2}$, as $T=\pi$ is the period of the infinitesimal vertical libration orbit. Instead, the family $T=2 \pi / 3$ originates at a displaced Lagrange point $\mathrm{L}_{2}$. Some of the orbits are plotted in Fig. 8b. The value of $\beta$ for each orbit can be read from Fig. 8c, plotted against $z_{0}$.

This completes the overview of the different families of eight-shaped orbits, with fixed sail attitude. Additional families can be found for different values of the sail attitude, and this is the subject of the next section. 


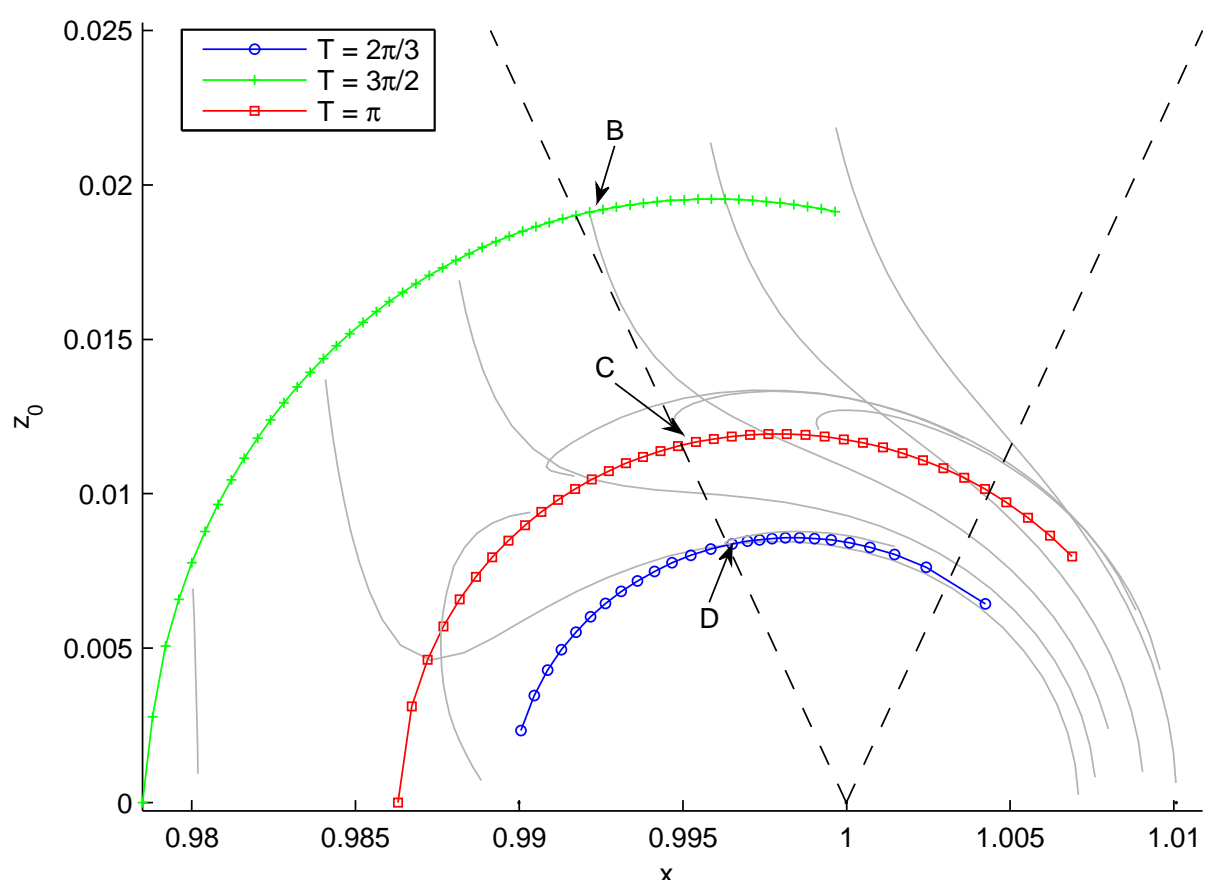

a)

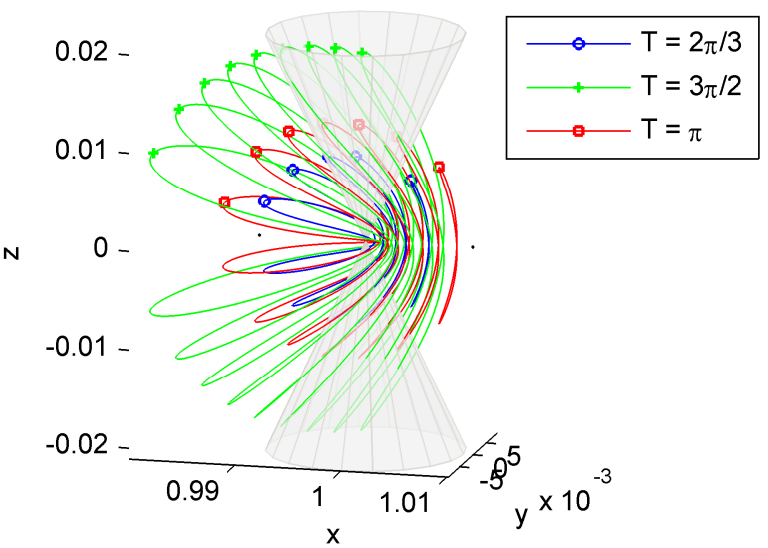

b)

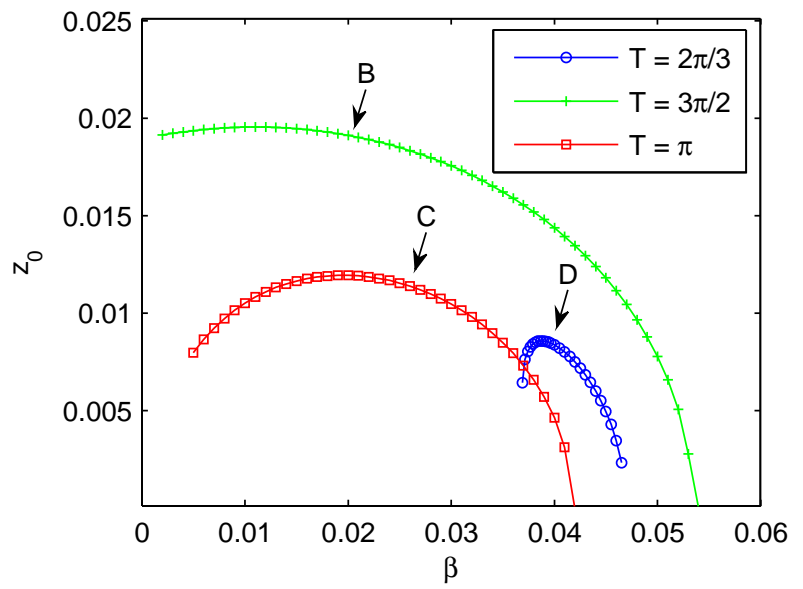

Fig. 8 Families of orbits with constant period $T$. (a) Initial points in the $x$-z plane. Gray lines represent other families of orbits presented in the paper, and are plotted as a reference; (b) Some of the orbits. (c) Lightness number $\beta$ versus $z$ amplitude 


\subsection{Tilted-sail orbits}

It is know from the literature (McInnes et al. 1994) that Lagrange points can be displaced out of the ecliptic plane by using a solar sail, if the attitude is such that a vertical component of force is generated. It was also shown (Waters,McInnes 2007) that orbits exist around these artificial equilibrium points, when the sail attitude is maintained fixed throughout the orbit. Interestingly, Farrés,Jorba (2010) highlighted that if the sail is tilted in the out-of-plane direction, one loop of a figure-eight becomes wider than the other. Therefore, we can envisage using these orbits for enhancing the visibility over one of the two hemispheres of the Earth. We consider tilting the vector $\hat{\mathbf{n}}$ by adding a $z$ component, and therefore keeping the vector in the $x-z$ plane. Again, within each orbit the sail attitude is kept fixed. This preserves the symmetry of the dynamical system with respect to the $x-z$ plane, while the symmetry with respect to the $x-y$ plane is obviously lost. Therefore, formally these orbits fall into the "type-A" category (the same as halo orbits).

Again we can exploit this symmetry for designing families of orbits. The family is found using the angle between the vector $\hat{\mathbf{n}}$ and the positive $x$-axis direction as a continuation parameter, increasing it of a small amount, step by step. Because the $x-z$ symmetry is retained, the initial state has still the form in Eq. (4). Again, the period is kept fixed but now the state at first intersection with $x-z$ plane cannot be used as a condition for solving the system. As for type-A orbits, fixing the period $T$, the initial state has to be integrated until $T / 2$, requiring that the state at this point is:

$$
\mathbf{s}_{2}=\left[\begin{array}{llllll}
x_{2} & 0 & z_{2} & 0 & v_{y 2} & 0
\end{array}\right]^{T}
$$

to preserve the symmetry about $x-z$. The three equations that will be used to determine $\mathbf{s}_{0}$ are then:

$$
\begin{aligned}
& y_{2}\left(x_{0}, z_{0}, v_{y 0}\right)=0 \\
& v_{x 2}\left(x_{0}, z_{0}, v_{y 0}\right)=0 \\
& v_{z 2}\left(x_{0}, z_{0}, v_{y 0}\right)=0
\end{aligned}
$$

It can be seen that, at constant lightness number and period, these points gradually displace vertically, then falling back towards the natural case, as the sail is tilted 
even more and the vector $\hat{\mathbf{n}}$ becomes almost aligned with the $z$ axis (and therefore the sail acceleration becomes negligible).

As a starting point for the continuation, any of the orbits presented in the previous section can be used, in principle. Only some representative families among all the possible orbits are presented here: four families with period $T=\pi$, one with $T=2 \pi / 3$. Fig. 9a shows once more the starting points in the $x-z$ plane; Fig. $9 \mathrm{~b}$ is a plot of some of the orbits; Fig. 9c allows reading the tilting angle of the sail, required by each orbit.

In particular, Fig. 9a clearly shows that, for the area of interest, the starting points of the orbits with a tilted sail are located at a $z_{0}$ higher than the corresponding point on the orbit with non-tilted sail. Fig. $9 \mathrm{~b}$ shows the actual shape change in the orbits due to the sail tilting. It can be noted that the orbits in the family lose their figure-eight shape as the sail is tilted, and they collapse into a simple orbit of half the period (multiplicity changes from 2 to 1 ). This makes these orbits less interesting for polar observation, and therefore they will not be considered. However, another more interesting effect is the fact that the time spent above the ecliptic on one of these orbits is longer than that spent below. This can be seen from the fraction of the orbit period on each orbit spent above the ecliptic, plotted in Fig. 9d. For some families, it can be consistently higher than $50 \%$, therefore it is reasonable to envisage the use of these orbits for continuous observation of the North Pole.

The next section will assess the visibility conditions that can be achieved considering five of the orbits presented here, indicated with the letters from A to E in the plots. 


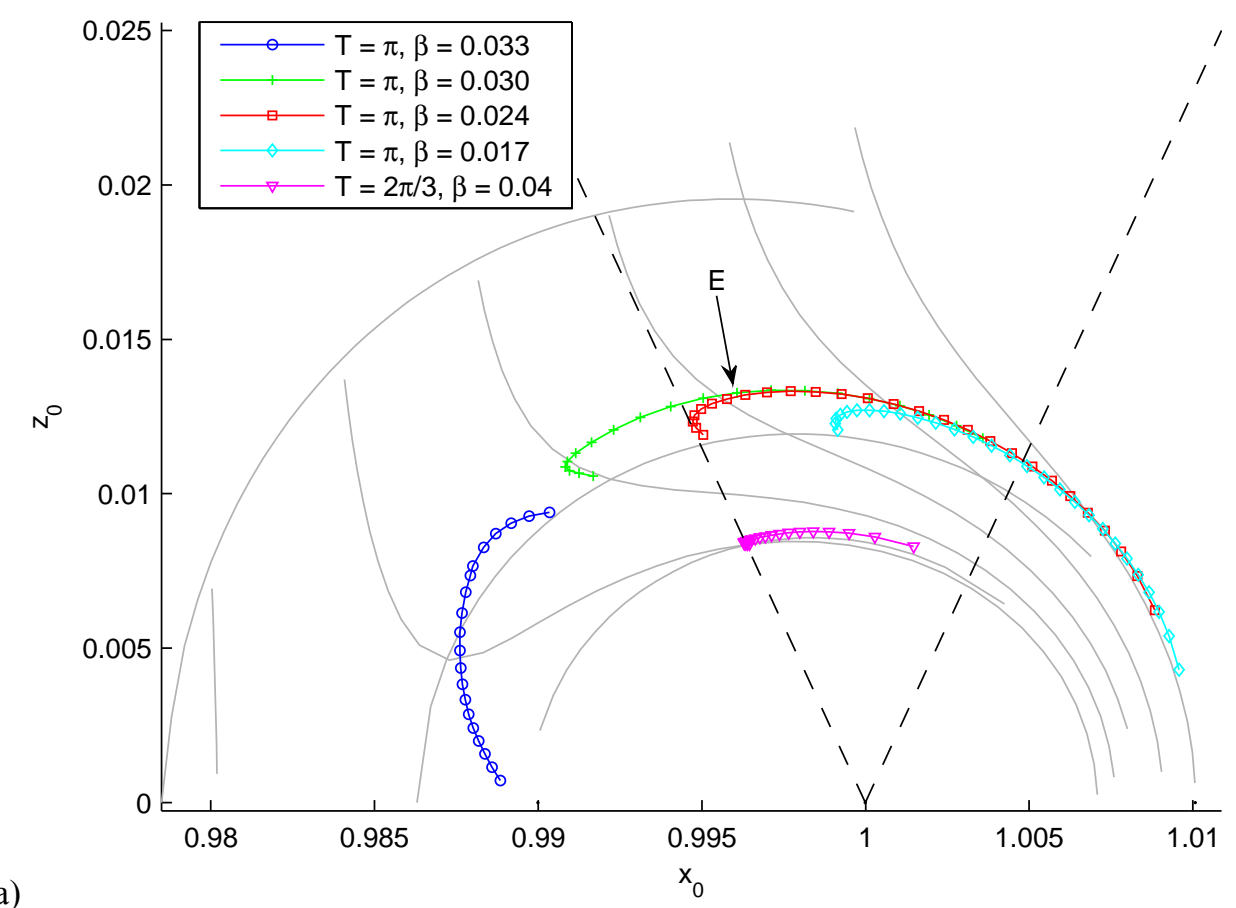

a)

b)
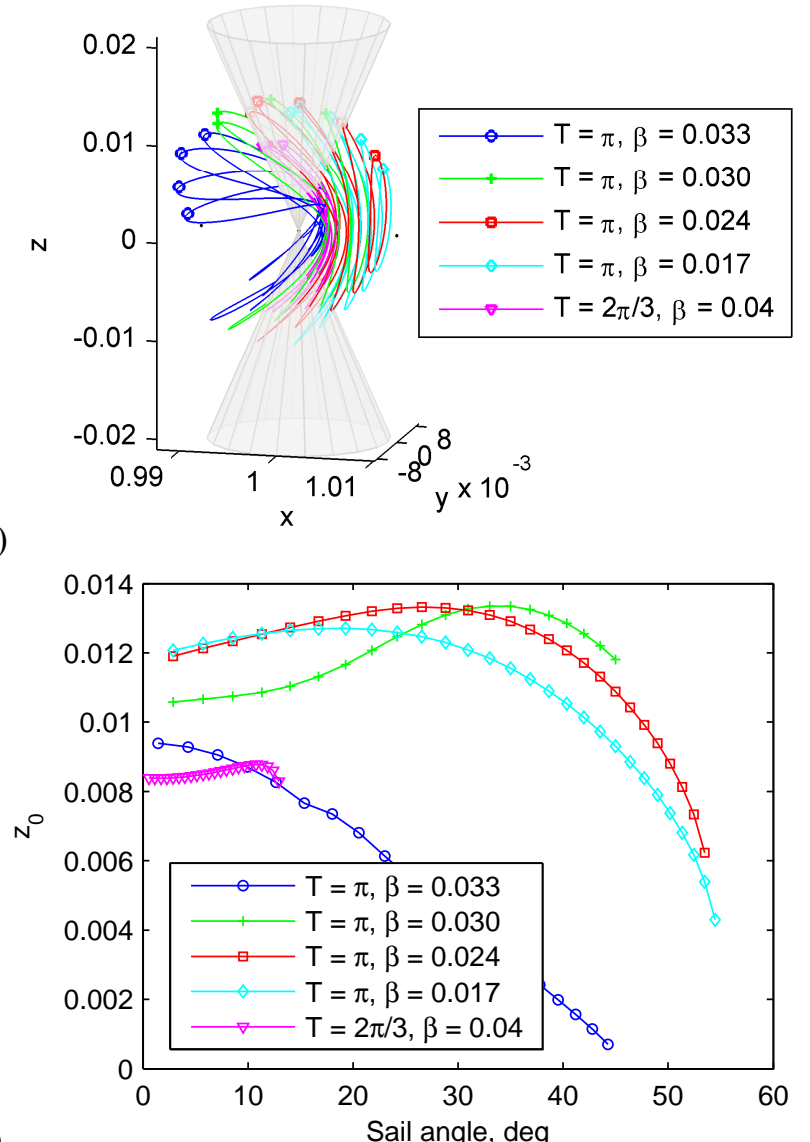

c) 


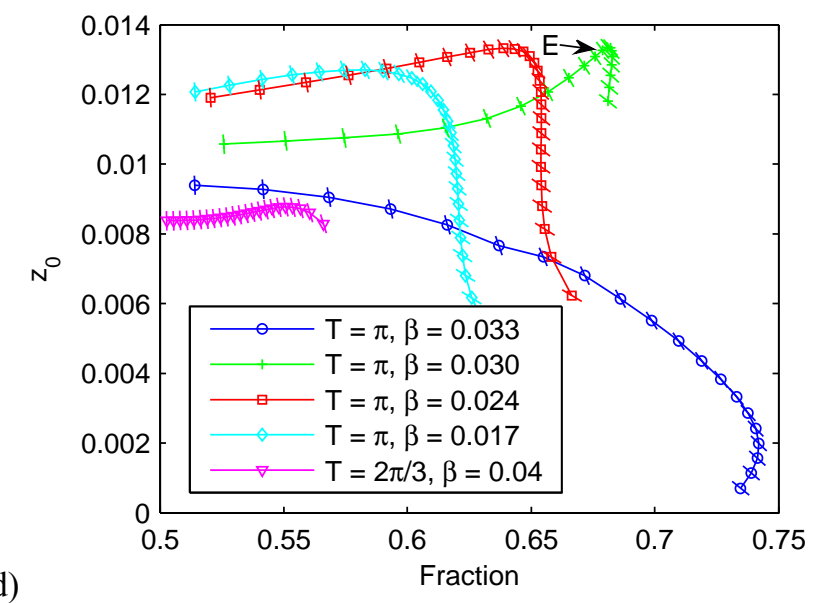

Fig. 9 Families of orbits with constant sail lightness number $\beta$ and period $T$. Within each family, the sail normal is gradually tilted towards the $z$ direction. (a) Initial points in the $x-z$ plane. Gray lines represent other families of orbits presented in the paper, and are plotted as a reference; (b) Some of the orbits. (c) Sail normal angle versus $z$ amplitude; (d) Fraction of the period spent above the ecliptic versus $z$ amplitude. The plot also shows graphically the sail orientation required (in an $x-z$ view) at different points in each family

\section{Visibility analysis}

It was noted that eight-shaped orbits do not allow a continuous view of one of the poles, as in the case of stationary spacecraft in the CR3BP or pole-sitters, due to their oscillation above and below the ecliptic plane. Therefore, a constellation of spacecraft is necessary to guarantee the coverage needed.

To assess the polar coverage provided by a spacecraft (or a constellation) in a displaced eight-shaped orbit, we will make use of two indicators: the first is the latitude $\varphi$ of the footprint of the spacecraft; in general, higher latitude implies better polar coverage. An ideal pole-sitter would constantly be at $\varphi=90^{\circ}$ (Ceriotti,McInnes 2011); however, in other types of orbits, the latitude changes throughout the mission and is therefore a function of time.

The second indicator is the latitude $\Lambda$, see Fig. 10. We define the access area as the spherical segment on the surface of the Earth that is accessible from the spacecraft with a minimum elevation angle $\alpha$, which is set to the reference value of $27^{\circ}$ (the elevation of a GEO spacecraft seen from $55^{\circ}$ latitude; this is the maximum latitude that is conventionally considered accessible from GEO). This segment is centered at the footprint of the spacecraft, and may include the pole of the Earth. If it does, then we can define the latitude $\Lambda$, which limits the spherical segment centered at the pole that the spacecraft can continuously access, despite the Earth's daily rotation around its polar axis (see Fig. 10). It can also be 
geometrically defined as the highest latitude of the bound of the access area, when the access area is including the pole.

Therefore, assuming the spacecraft is in the same position during a day (or its position changes only slightly), the green area in Fig. 10 would be accessible at any time during that day, while the yellow area cannot see the spacecraft for some period due to the rotation of the Earth. Consequently, all the latitudes on the Earth above $\Lambda$ can continuously access the spacecraft with a minimum elevation of $\varepsilon$ at any time of day.

It is desirable to have a low (in modulus) value of $\Lambda$, as this would mean continuous coverage not only of the polar caps, but also lower-latitude regions around the pole, and therefore the spacecraft can observe not only to the pole, but also lower latitudes of the Earth.

Note that the angle $\Lambda$ depends not only on the spacecraft latitude $\varphi$, but also on its distance from the Earth. Also, if the instant access area does not cover the pole, then there is no continuous access area, and $\Lambda$ cannot be defined. This can happen when the latitude $\varphi$ of the spacecraft is so low (with respect to its distance) that no continuous coverage is possible at any latitude.

It should be noted that the relative position of the spacecraft with respect to the Earth changes, and the angle $\Lambda$ in general varies during each eight-shaped orbit. In this case, its maximum (in absolute value) represents the worst case. Finally, if a constellation of spacecraft is considered, then the value of $\Lambda$ to be considered at any given time is the lowest (in magnitude) of each one of the spacecraft. 


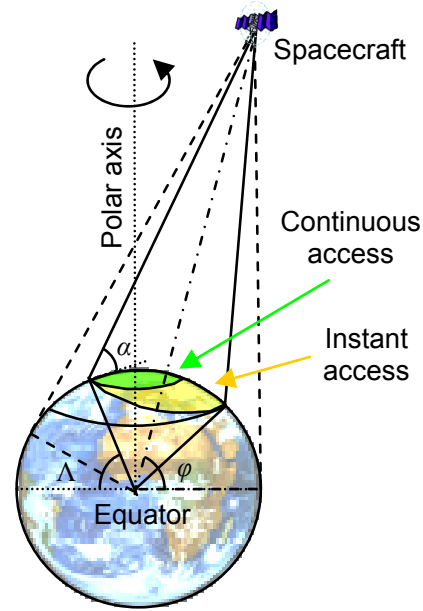

Fig. 10 Geometry of the spacecraft coverage. The yellow area is the surface accessible with minimum elevation angle of $\alpha$, instantaneously. The green area is the surface that is continuously accessible from the spacecraft with the same minimum elevation angle: therefore $\Lambda$ is the minimum latitude of continuous coverage

We will now consider the five orbits indicated with the letters $A$ to $E$ in the previous figures, and study the visibility in terms of $\varphi$ and $\Lambda$ throughout a year: in fact, for all the cases considered, the relative position of the formation is oneyear periodic.

In all the mission scenarios, a constellation of two or three spacecraft, equally spaced in time on the orbit, is used. The initial position of the spacecraft formation is chosen such that one spacecraft is at the northern intersection with the $x-z$ plane at northern summer solstice. For the selected orbits, this choice optimizes the visibility conditions of the northern pole while it is illuminated.

Moreover, in the plots, the time $t=0$ corresponds to the northern winter solstice, i.e. $21^{\text {st }}$ December, when the northern half of the polar axis of the Earth leans away from the Sun, and the South Pole is illuminated.

The first orbit, A, is a natural orbit, with period $T=3 \pi / 2$ (9 months). It is then the intersection between the two families $\beta=0$ and $T=3 \pi / 2$. Note that the northern apex of the orbit lies above the Earth (although it is never aligned with its polar axis). This orbit is chosen because it is a good compromise between visibility and repeat time, and has its main advantage in the fact that it does not need any low-thrust acceleration. Therefore it does not need a solar sail, and in general any propulsion system, apart from the control that might be required to counteract perturbations and orbit instability. 
A minimum of three spacecraft are necessary for guaranteeing continuous visibility of both poles at any time of the year, i.e. at any time both poles can be accessed by at least one spacecraft in the constellation. The visibility that a constellation on this orbit can offer is shown in Fig. 11. It can be seen that the highest $\Lambda$ during the mission is of order $74^{\circ} \mathrm{N}$ for the North Pole and $76^{\circ} \mathrm{S}$ for the South Pole. The northern case happens twice a year in May and August, while the southern case happens once on $21^{\text {st }}$ December.
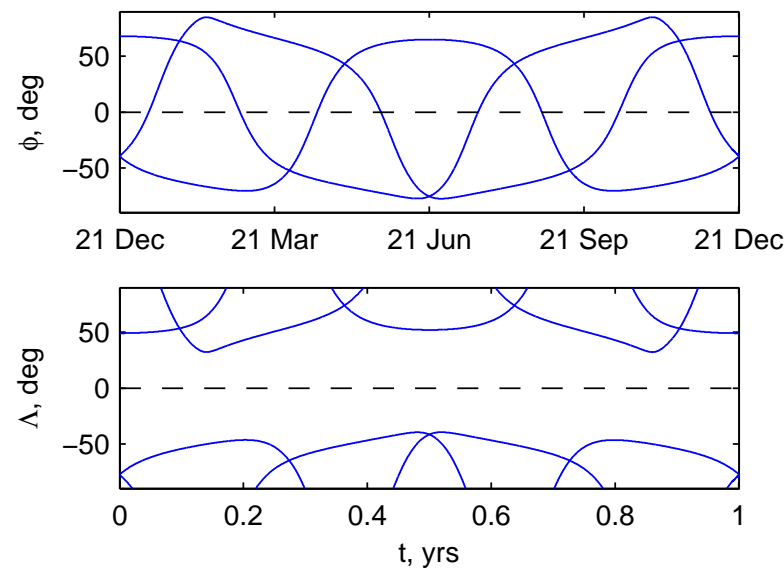

Fig. 11 Latitude and visibility of three spacecraft on natural orbit $\mathrm{A}(\boldsymbol{\beta}=\mathbf{0}, T=3 \pi / 2)$

By adding a solar sail, it is possible to move on the $T=3 \pi / 2$ family and therefore select an orbit whose starting point is close to the position of the polar axis of the Earth on the $21^{\text {st }}$ June (northern summer solstice). This allows a spacecraft on this orbit to follow closely the polar axis for a longer period (see again Fig. 8b). The selected orbit is that with $\beta=0.02$, and therefore it is once again at the intersection of the two families in the previous figures (identified with B). The visibility of three spacecraft on this orbit is represented in Fig. 12.

From the plots, the advantage of selecting an orbit that reaches the polar axis of the Earth on the Sun side is evident: one spacecraft is exactly above the North Pole at northern summer (and hence with favorable lighting), and also the spacecraft are high in the sky on the South Pole during southern summer. The configuration of this orbit, then, is ideal for polar observation in particular in the visible range, when either pole is illuminated by the Sun. 

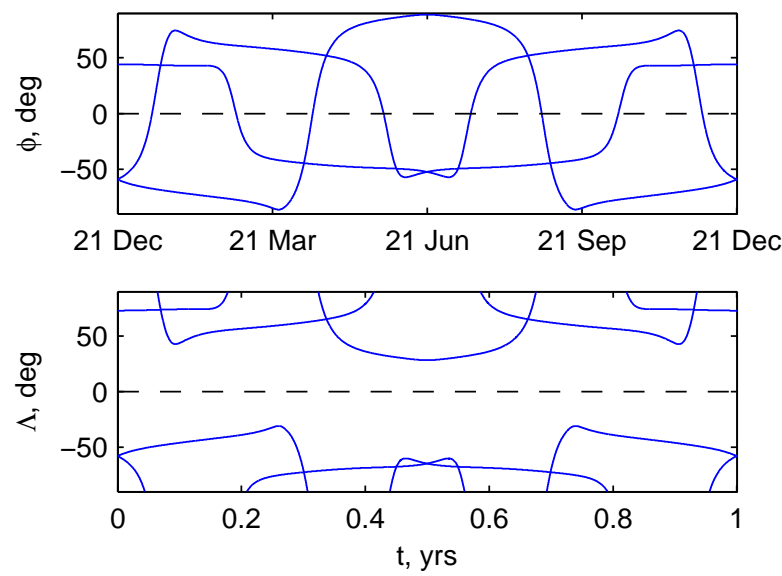

Fig. 12 Latitude and visibility of three spacecraft on orbit $B(T=3 \pi / 2, \beta=0.02$, sail normal along $x$ )

The main drawback of both orbits A and B presented above is the considerable distance from the Earth (the distance of the orbit apex from the Earth can be seen in Fig. 8). Reducing the period of the orbits allows the spacecraft to be closer, at the cost of having shorter intervals in which each spacecraft observes one pole continuously. We consider the family of orbits with $T=\pi$ (6 months) and again the orbit in which the starting point is aligned with the polar axis on the Sun side, which results in $\beta=0.026$ (see Fig. 13). This is the orbit identified as $\mathrm{C}$ previously. Even in this case, the visibility conditions are better at the pole that is illuminated, however the shorter period of the orbit enforces more frequent switching points (6 every year) for continuous polar observation.

For this orbit, the maximum $\Lambda$ is of order $77^{\circ} \mathrm{N}$ on $21^{\text {st }}$ December for the North Pole and $75^{\circ} \mathrm{S}$ twice a year for the South Pole, around end of April and beginning of July.
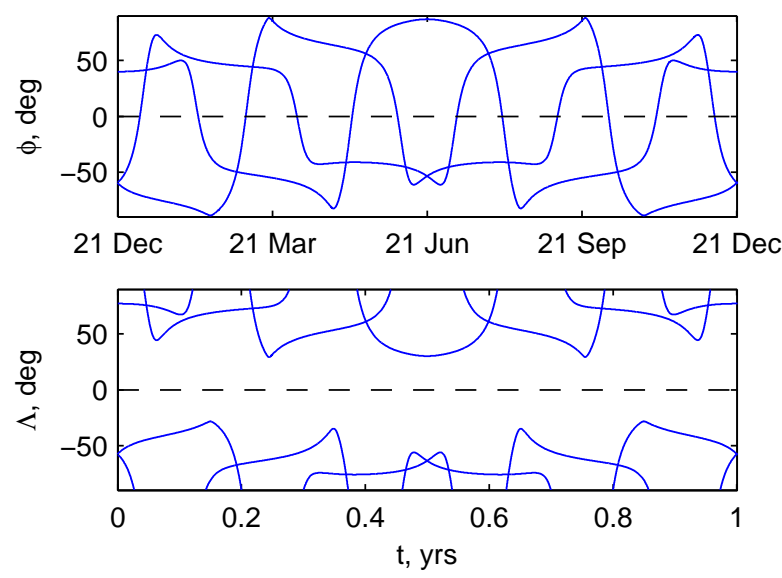

Fig. 13 Latitude and visibility of three spacecraft on orbit $\mathrm{C}(T=\pi, \beta=\mathbf{0 . 0 2 6}$, sail normal along $x$ ) 
The spacecraft can observe the poles even closer by selecting an orbit in the family $T=2 \pi / 3$ (equivalent to 4 months), although even more switching points are necessary ( 9 in a year). The orbit that crosses the polar axis at solstices is that with $\beta=0.04$, which is marked with $\mathrm{D}$ and its visibility conditions are plotted in Fig. 14. For this case, the maximum $\Lambda$ is of order $73^{\circ}$ for both North and South Poles at solstices.
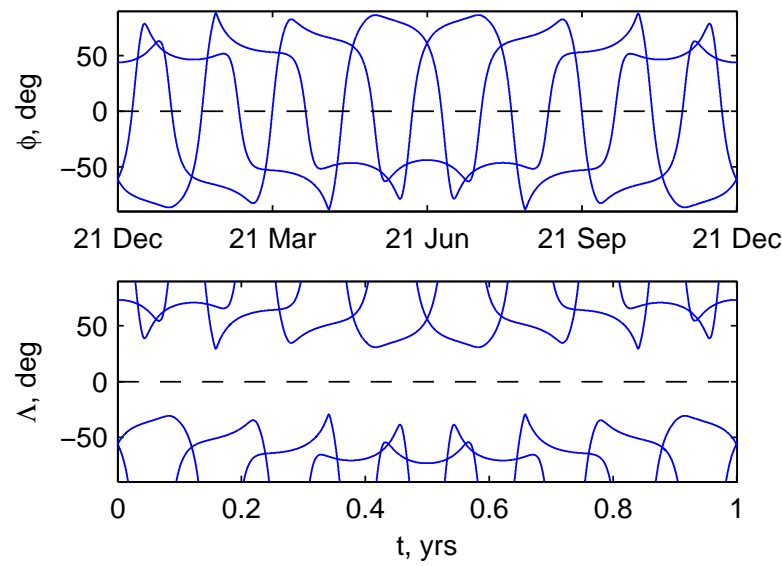

Fig. 14 Latitude and visibility of three spacecraft on orbit $D(T=2 \pi / 3, \beta=0.04$, sail normal along $x$ )

The last orbit we consider is one obtained by tilting the sail normal northwards, and therefore displacing the orbit above the ecliptic. As noted earlier, since the orbit is not symmetric to the ecliptic plane, it shall be used for having better visibility conditions over one of the poles, specifically the North Pole when the sail normal is tilted towards the positive $z$ axis. Looking again at the plots in Fig. 9, we can see that the family $T=\pi, \beta=0.03$ is the one that offers the highest increase in fraction of the period spend above the ecliptic, while maintaining appropriate $z$ amplitude. The family $T=\pi, \beta=0.033$ spends a longer fraction of a period above the ecliptic, but its out-of-plane displacement is such that it cannot be used for polar observation. Conversely, the other two families do not benefit as much from tilting the sail.

Within the family we select the orbit identified with E in Fig. 9, as it provided the best compromise in terms of visibility, because it is close to the orbit that maximizes the fraction of period above the ecliptic (see Fig. 9d) while at the same time being close to the polar axis on the Sun side. 
Since it was shown three spacecraft are sufficient to observe both poles, then we shall investigate if it possible to observe continuously one pole with two spacecraft only: plots of $\varphi$ and $\Lambda$ are shown in Fig. 15 .

The minimum latitude $\varphi$ of either spacecraft is $50^{\circ}$, and the continuous visibility latitude $\Lambda$ is $66^{\circ}$ in the worst case, twice per year, before and after northern summer solstice.

The advantage of this orbit with respect to using the same number of spacecraft in a Molniya-type orbit is a much longer period of each spacecraft over the pole, therefore reducing consistently the required switching and offering a platform for continuous observation with intervals of about 3 months, rather than a few hours. The drawback is of course the increased distance at which observations are made.
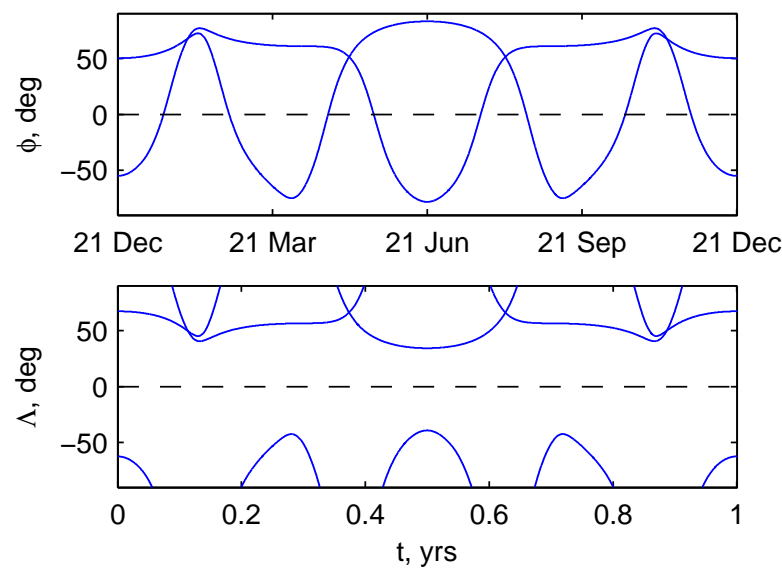

Fig. 15 Latitude and visibility of two spacecraft on orbit $E(T=\pi, \beta=0.03, n=[0.8575,0$, $0.5145]^{T}$ corresponding to a tilting of $30.96^{\circ}$ w.r.t. the positive $x$ axis)

\section{Stability}

Stability is a desirable property of science orbits, which need to be maintained for an extended period of time. If an orbit is stable, then in principle no active control is needed to maintain the spacecraft on that orbit. Conversely, for an unstable orbit, an infinitesimal perturbation in the direction of the unstable eigenvector can cause the spacecraft to depart from that orbit indefinitely.

It is known that collinear equilibrium points are unstable, therefore it is expected that infinitesimal orbits around these points are unstable as well. However, as the orbit amplitude increases, higher-than-linear order terms start to become significant and can change the stability properties of the orbit. For example, Baig,McInnes (2009) showed that, despite that small-amplitude displaced halo 
orbits are unstable, as the amplitude increases, a range of stable orbits is found. We therefore investigate here if the same happens for natural and displaced eightshaped orbits.

To assess the stability of a periodic solution of a dynamical system, we make use of the Floquet theory.

Given a non-linear first-order dynamical system of differential equations, $\dot{\mathbf{s}}=\mathbf{f}(\mathbf{s})$, such as Eq. (3), let $\mathbf{s}^{*}(t)$ be a periodic solution of period $T$, such that: $\mathbf{s}^{*}(t)=\mathbf{s}^{*}(t+T)$. By introducing a small perturbation on the states $\delta \mathbf{s}$, and expressing a general solution as $\mathbf{s}=\mathbf{s}^{*}+\delta \mathbf{s}$, we can linearize the system around the periodic solution:

$$
\delta \dot{\mathbf{s}}=\left.\frac{\partial \mathbf{f}}{\partial \mathbf{s}}\right|_{\mathbf{s}=\mathbf{s}^{*}} \delta \mathbf{s}
$$

The matrix $\left.\frac{\partial \mathbf{f}}{\partial \mathbf{s}}\right|_{\mathbf{s}=\mathbf{s}^{*}} \equiv \mathbf{A}^{*}(t)$ is simply the Jacobian $\mathbf{J}=\partial \mathbf{f} / \partial \mathbf{s}$ of the system evaluated along the periodic solution, and therefore it is a function of time. Eq. (17) can be written as a non-autonomous, periodic linear system, that represents the variations in the vicinity of $\mathbf{s}^{*}$ :

$$
\delta \dot{\mathbf{s}}=\mathbf{A}^{*}(t) \delta \mathbf{s}
$$

The state transition matrix $\boldsymbol{\Phi}(t)$ is defined as the solution of the differential system:

$$
\dot{\boldsymbol{\Phi}}=\mathbf{A}^{*}(t) \boldsymbol{\Phi}, \quad \boldsymbol{\Phi}(0)=\mathbf{I}
$$

and allows us to find the states of the system after some time $t$ when an arbitrary small perturbation is applied to $\mathbf{s}^{*}(0)$. The state transition matrix evaluated at exactly one period $T$ is the monodromy matrix, $\mathbf{M} \equiv \boldsymbol{\Phi}(T)$. According to Floquet theory (Thurman,Worfolk 1996), the first order linear stability properties of a periodic orbit are described by the eigenvalues of the monodromy matrix $\mathbf{M}$. The Jacobian A can computed analytically for the system in Eq. (3), and it can be shown that $\operatorname{tr}(\mathbf{A})=0$ : therefore, it can be demonstrated through Louiville's theorem that the eigenvalues of the monodromy matrix occur in reciprocal pairs (Broucke et al. 1976). In particular, the orbit is stable when all three pairs of eigenvalues lie on the unit circle. Instead, the orbit is unstable if there is pair of real eigenvalues: 


$$
\begin{aligned}
& \lambda_{1}=\lambda_{r} \\
& \lambda_{2}=1 / \lambda_{r}
\end{aligned}
$$

such that $\lambda_{r} \geq 1$. Therefore to assess the stability of the orbits, we can monitor the magnitude of $\lambda_{r}$, being stable if it is equal to 1 , or unstable if greater than one. Fig. 16 shows the magnitude of $\lambda_{r}$ for each orbit in the families identified earlier, plotted as a function of the initial state $z_{0}$ of each orbit. It is found that that all the orbits are unstable, as $\lambda_{r}>1$. However, the figure also shows that the sail has, in some circumstances, a beneficial effect on the stability of the orbits. For example, let us observe the families with constant $\beta$ in Fig. 16a. It is clear that for a certain range of amplitudes, the maximum eigenvalue magnitude becomes smaller than 10 , being up to three orders of magnitude smaller than the one of an orbit with the same amplitude, but no solar sail. The same effect is visible in the other two plots of the figure: in Fig. 16b, the orbits with lowest maximum eigenvalue magnitude are those with higher values of $\beta$ (compare with Fig. 8c), and in Fig. 16c, the family with $\beta=0.033$ has the best stability values, even if its $z$-amplitude is small. Also note that, in Fig. 16a, only the orbits in the family $\beta=0.05$ with $T<3 \pi / 2$ are plotted, consistently with Fig. 7a. 


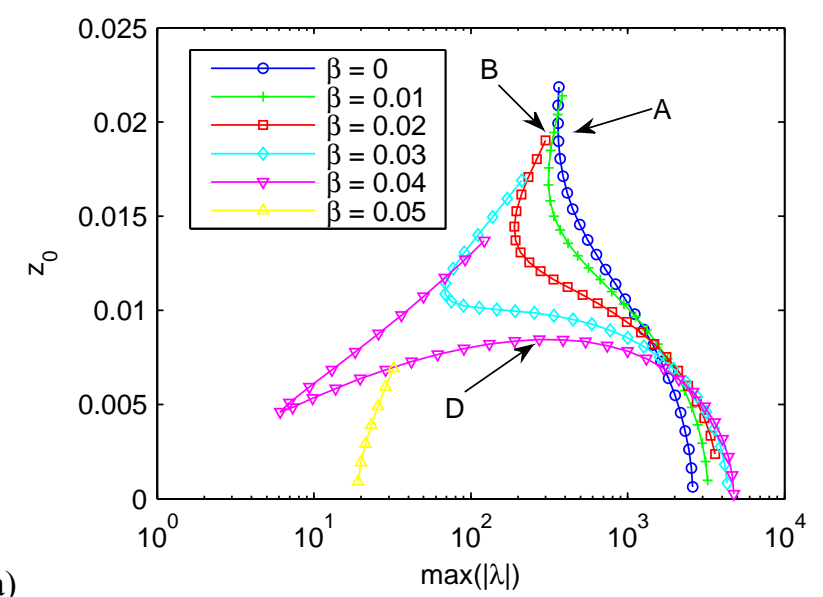

a)

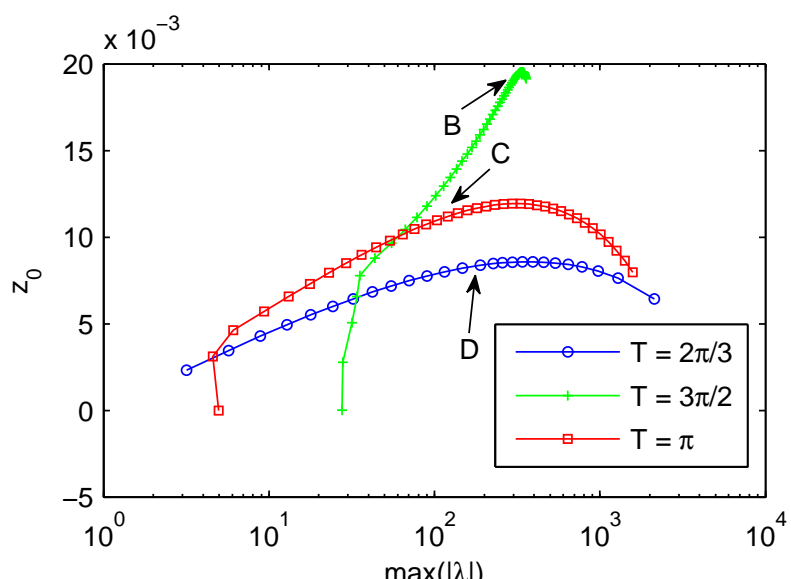

b)

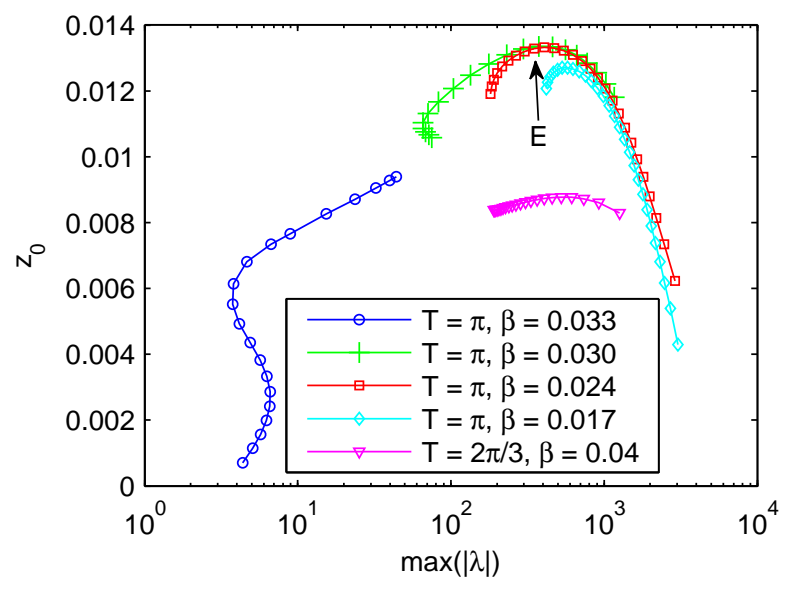

Fig. 16 Magnitude of the maximum eigenvalue of the monodromy matrix (in logarithmic scale), as function of the $z$ amplitude. (a) Families of orbits with constant $\beta$; (b) Families of orbits with constant $T$; (c) Families of orbits with constant $T$ and $\beta$, varying the sail tilting

Limiting our analysis to the families with sail perpendicular to $x$, and in order to provide a better understanding of their stability varying the $\beta$ values, we consider here three sets of orbits in which the $z$-amplitude (i.e. $z_{0}$ ) is fixed, and the value of the lightness number is varied through the family with a constant step, from 0 to 0.05 . This corresponds to a set of starting points that define a horizontal segment in Fig. 7a. As before, we follow the evolution of the eigenvalue $\lambda_{r}>1$, along each family, as an indicator of the stability, and this is plotted for three 
values of $z_{0}$ in Fig. 17. These plots highlight that for example, along the family $z_{0}=0.01$, the orbits have a minimum of $\lambda_{r}$ at $\beta \cong 0.036$ and $x_{0} \cong 0.987$ (with period $T \cong 3.815$ ). Therefore, although stability is never reached, the highly unstable nature of eight-shaped orbits is mostly reduced around this value of the lightness number. As the $z$-amplitude increases, the variation of $\lambda_{r}$ along the families becomes less sensitive, as can be seen from the cases $z_{0}=0.015$ and $z_{0}=0.02$.

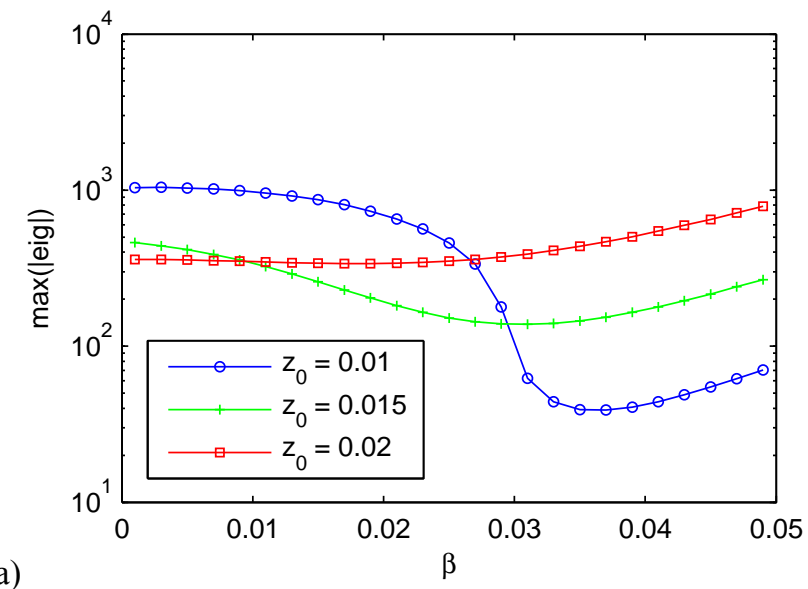

a)

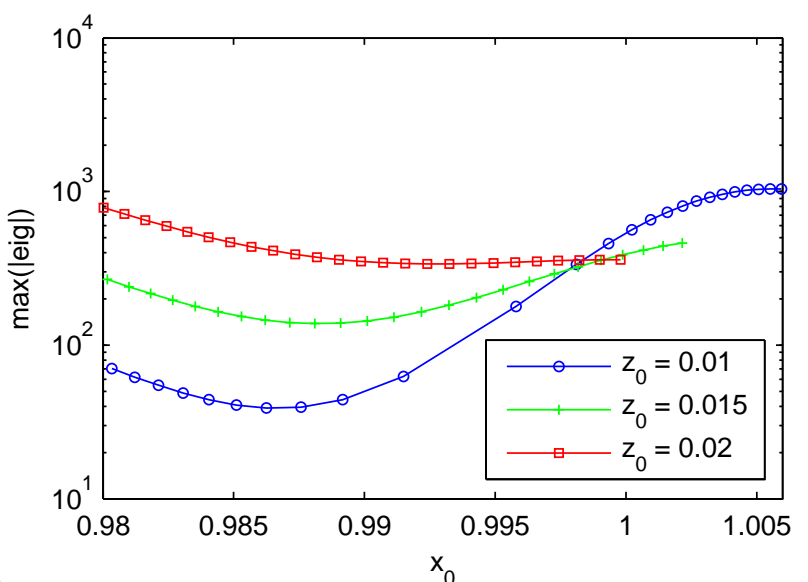

b)

Fig. 17 Magnitude of the maximum eigenvalue of the monodromy matrix (in logarithmic scale) for families of orbits with constant $z$-amplitude. (a) As a function of the lightness number; (b) As a function of $x_{0}$.

\subsection{Orbit control}

Since all the orbits resulted to be linearly unstable, a spacecraft would necessarily need a form of active feedback control, in order to stay on these orbits for a considerable amount of time. Devising a complete, optimal control strategy for these kinds of orbits is beyond the scope of this paper. Nonetheless, it is 
interesting to assess the difficulties which might arise when a control strategy is put in practice.

The purpose of a feedback control is to provide a steering law for the solar sail which essentially guarantees that the spacecraft stays in the vicinity of the reference solution even when orbit instability, external perturbations and displacements from initial conditions are taken into account.

In order to have a preliminary estimation of it, we designed and implemented an LQR. The controller is based on the work developed by Ceriotti,McInnes (2010); however, in that work, an additional thruster was used for the control of the spacecraft. Here, we would like to assess the controllability of the spacecraft by using the solar sail only.

Let us consider again the dynamics of the solar sail in the three-body problem as described by Eq. (3), in which the solar sail attitude and lightness number are used as control parameters $\mathbf{u}$, and let us consider a solution of it $\mathbf{s}(t), \mathbf{u}(t)$ that will be used as a reference, to be tracked by the spacecraft. In all the orbits presented before, the solar sail has constant attitude and lightness number, therefore the reference control vector is simply constant with time.

In general, when the dynamics is integrated in time starting from the initial conditions $\mathbf{s}(0)$ and the control law $\mathbf{u}(t)$, the spacecraft will depart from the reference states $\mathbf{s}(t)$, due to the instabilities of the orbit. Let us define the state error $\delta \mathbf{s}$ as the difference between the reference state and the real state at any given time. Assuming that the real state is not too far from the reference one, and that the evolution of the dynamics of the system is slow enough, then at each instant of time the system can be linearised around the reference state and control, obtaining:

$$
\delta \dot{\mathbf{s}}=\mathbf{A} \delta \mathbf{s}+\mathbf{B} \delta \mathbf{u}
$$

This system represents the variations around the reference state and control at any given time, and $\delta \mathbf{u}(t)$ is the feedback control (to be determined), i.e. the quantity that, when added to the reference control $\mathbf{u}(t)$, will bring the spacecraft back to reference conditions (within linear and time-invariant approximation). If we introduce the functional:

$$
J=\int_{0}^{\infty}\left(\delta \mathbf{s}^{T} \mathbf{Q} \delta \mathbf{s}+\delta \mathbf{u}^{T} \mathbf{R} \delta \mathbf{u}\right) d t
$$


then the minimum of this functional guarantees that the feedback control strategy $\delta \mathbf{u}(t)$ is such that both the state error and the feedback control at infinity are zero, and therefore the spacecraft is back to the reference conditions.

We now assume a feedback control proportional to the state error:

$$
\delta \mathbf{u}=-\mathbf{K} \delta \mathbf{s}
$$

It can be shown that the minimum of functional (19) can be found explicitly by solving the algebraic Riccati equation (Bryson,Ho 1975), which provides the optimal values of the gain matrix $\mathbf{K}$.

Note that, despite the linear system is time-invariant, the linearisation and the solution of the Riccati equation is performed at each instant of time, therefore resulting in a time-variant gain matrix.

In the control method implemented in this work, the vector $\mathbf{u}$ contains two Cartesian components of the unit vector $\hat{\mathbf{n}}$ in the plane perpendicular to the sun line (this choice is made to avoid singularities), and the lightness number $\beta$ of the solar sail, for a total of three control variables.

Steering a solar sail is a standard control method, which has been investigated in the literature extensively. In addition to the actuators commonly used on conventional spacecraft (inertia wheels, thrusters), sail steering can also be achieved exploiting the sail itself. Several methods were proposed, including shifting of the center of mass with respect to the center of pressure through moving masses (Lappas et al. 2005), deployment of control vanes at the edge of the sail (Wie 2004), and also differential change of reflectivity on opposite sides of the sail through photo-chromic areas (Funase et al. 2010). These areas are covered with a special material that can change its reflectivity coefficient within a given range according to a current that is applied to it.

The change of lightness number can essentially be achieved in two ways: varying the area of the sail or its reflectivity. The first method would make use of the same reflecting control vanes used for attitude control, but they would be deployed symmetrically; the latter method would make use of the photo-chromic area on the sail, again changing their reflectivity symmetrically. This control method was implemented in MATLAB/Simulink and applied to the orbits B, C, D (orbit A has no sail), and it stabilized the system with small control effort. We also assessed the capability of the controlled system to come back to the reference conditions, when the initial state is perturbed. This can be 
interpreted as a step response of the controlled system, when the step is applied at time $t=0$, and therefore at the northern apex of the orbits. We considered displacements in one component of the initial state vector, of $10^{-4}$ in position or $10^{-3}$ in velocity. This case models, for example, an error at the injection point. For the three mentioned orbits, the control guarantees that the system converge to reference conditions. The amount of time needed to bring the state error below a given threshold depends obviously not only on the gain matrices, but also on the reference orbit, and it may require more than one orbital period. Fig. 18 shows the results for orbit $\mathrm{B}$ when the $x$-component of the initial state is displaced by $10^{-4}$. The two plots in Fig. 18a show the state error (position and velocity), and it is clearly seen the initial error in position, and the convergence to reference values within two orbital periods. Note that in order to correct the position, a transient error in the velocity has to be introduced. The two plots in Fig. 18b show the control effort needed; in particular, the top plot shows the angle between the sail normal when feedback control is added, and the reference (i.e. along the $x$-axis). Note that this does not completely define the sail normal direction, however it is a measure of the tilting necessary to control the spacecraft, and therefore the control effort. The bottom plot in Fig. 18b shows the controlled lightness number (the dashed line is the reference - constant - lightness number). These two plots highlight that a tilting of less than 2 deg and a small change in reflectivity are sufficient to control the system, in presence of the step disturbance assumed before. Finally, Fig. 18c is a plot of the controlled trajectory (blue line), the reference orbit (red line), and the uncontrolled trajectory, i.e. the result of the integration of the equations of motion for one orbital period, without using the feedback control, and starting from the same displaced initial conditions. It is clear that the spacecraft diverges from the reference states well before one orbital period. 


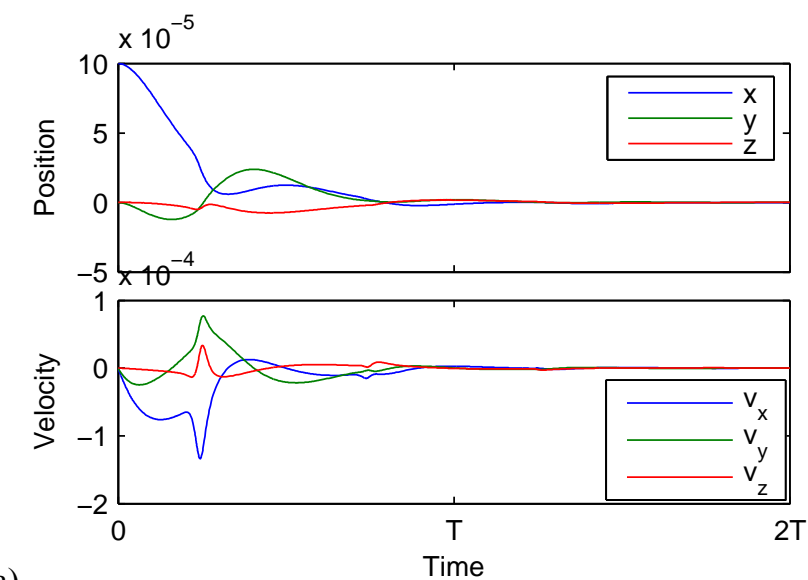

a)
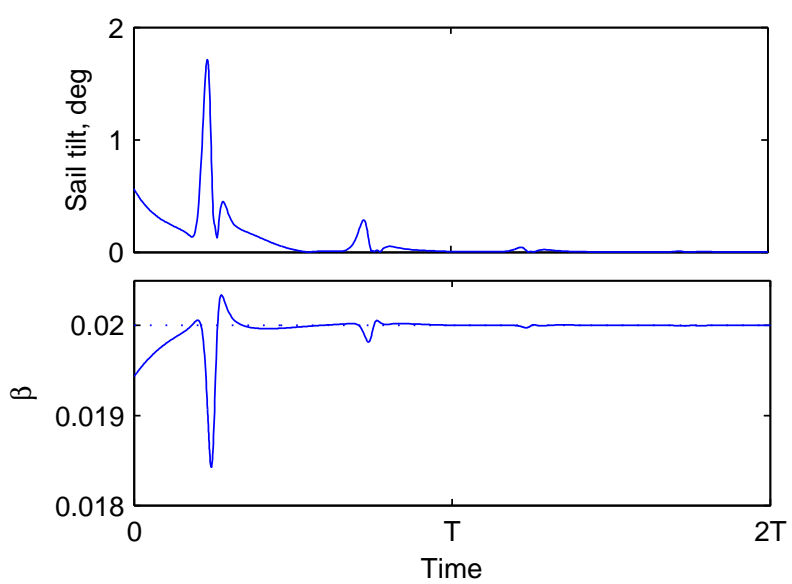

b)

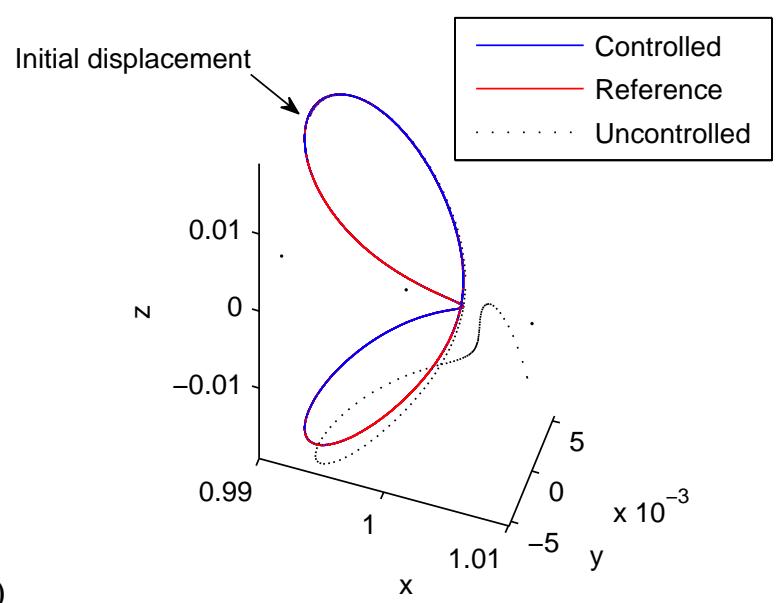

Fig. 18 Feedback control applied to orbit B. (a) State error (reference with respect to real); (b) Sail normal tilting with respect to the reference and controlled lightness number (reference dashed); (c) Controlled trajectory, reference and uncontrolled trajectory after one period.

A less advanced control method would involve the change in attitude only, with no change in sail reflectivity. This method would not need control vanes, but just tilting masses on the spacecraft bus. Although it was found that the linear system is still controllable (in terms of the controllability matrix), it was not possible to find weights such that the control loop stabilized the non-linear dynamics on a 
wide range of reference orbits. It is suspected that the non-linear dynamics of the eight-shaped orbits is too fast near the intersection point with the $x$-axis for the time-invariant approximation to be valid. A non-linear, time-variant control might be able to stabilize the system. Another type of sail control, based on the study of the manifolds around a halo orbits, was demonstrated successful in the literature and will be briefly presented in the following section.

\section{Invariant manifolds}

The instability of the presented orbits implies the existence of unstable invariant manifolds, which can be exploited for low-energy transfers to those orbits. Invariant manifolds associated with a periodic orbit are surfaces (in the state space) generated by natural trajectories that wind from or onto a periodic orbit. These trajectories are found by integrating an initial condition, obtained by perturbing the state vector at any point along the orbit, in particular directions. The directions are found by linearizing the system, and computing the eigenvalues and eigenvectors of the Jacobian matrix at a given time and state along the periodic orbit. The eigenvector associated with the real eigenvalue $\lambda_{1}>1$ gives the unstable direction. The eigenvector associated with the real eigenvalue $\lambda_{2}<1$ gives the stable direction.

A first-order approximation for the initial conditions of the manifold can be found using the following procedure, which is summarized here and explained in detail by Koon et al. (2006).

We find the manifold at time 0 and state $\mathbf{s}_{0}=\mathbf{s}(0)$ of the periodic orbit. First, we compute the state transition matrix over the orbit $\boldsymbol{\Phi}(t)$, and the monodromy matrix $\mathbf{M}=\boldsymbol{\Phi}(T)$, as detailed earlier. Then, we compute the eigenvalues of this matrix, and identify the real couple $\lambda_{1}>1, \lambda_{2}<1$ and associated eigenvectors, $\mathbf{v}_{0}^{u}$ and $\mathbf{v}_{0}^{s}$. The linear approximations of the initial states on the unstable $\left(\mathbf{s}_{0}^{u}\right)$ and stable $\left(\mathbf{s}_{0}^{s}\right)$ manifolds are given by:

$$
\begin{aligned}
& \mathbf{s}_{0}^{u \pm}=\mathbf{s}_{0} \pm \varepsilon \frac{\mathbf{v}_{0}^{u}}{\left|\mathbf{v}_{0}^{u}\right|} \\
& \mathbf{s}_{0}^{s \pm}=\mathbf{s}_{0} \pm \varepsilon \frac{\mathbf{v}_{0}^{s}}{\left|\mathbf{v}_{0}^{s}\right|}
\end{aligned}
$$


where the $+/$ - denote the two possible directions of the perturbation. Here $\varepsilon$ is a small positive number.

Finally, the manifolds can be computed at any time $t=\tau$ on the periodic orbit simply by transporting the stable and unstable directions through the state transition matrix, such that:

$$
\mathbf{v}^{u}(\tau)=\boldsymbol{\Phi}(\tau) \mathbf{v}_{0}^{u}
$$

and the same for the stable direction.

A note on the magnitude of $\varepsilon$ : it shall be small enough to fall into the linear approximation of the system, however not too small otherwise the time of flight becomes too large (Koon et al. 2006). In this paper $\varepsilon=200 \mathrm{~km}$ was used (Howell et al. 1994).

By integrating $\mathbf{s}^{u+}$ and $\mathbf{s}^{u-}$ forward in time using the full equations of motion, we obtain two trajectories that wind off the periodic orbit. The same behavior is obtained integrating $\mathbf{s}^{s-}$ and $\mathbf{s}^{s+}$ backward in time. Instead, if $\mathbf{s}^{u+}$ and $\mathbf{s}^{u-}$ are integrated backward in time, the trajectory winds onto the periodic orbit; the same behavior is obtained integrating $\mathbf{s}^{s-}$ and $\mathbf{s}^{s+}$ forward in time.

Therefore, we can exploit the stable manifolds for designing transfers to the periodic orbit; assume that stable manifolds are designed along the whole periodic orbit, and integrated backward in time, find a number of trajectories that wind off the periodic orbit. If the spacecraft is injected onto one of these trajectories (forwards in time), the method guarantees that it will asymptotically reach the periodic orbit without any additional maneuvers.

Particularly interesting are those unstable manifold trajectories that, when integrated backwards, pass the vicinity of the Earth, at a distance that is comparable to low Earth orbit. This is because the spacecraft can be injected directly onto the manifold from Earth, for example using a launcher upper stage, without the need for any additional deep space maneuvers. This procedure was used to find transfer trajectories to halo orbits (Gómez et al. 1993). Howell et al. (1994) showed that, if a manifold passes close enough to the Earth, then a very small maneuver applied at the insertion into periodic orbit is sufficient to change the minimum distance to the Earth, and tune it for a selected altitude. This can be achieved with a simple predictor-corrector method. Alternatively, low-thrust 
propulsion has also been used to find optimal transfers to eight-shaped orbits (Senent et al. 2005).

In this paper, we will only study the manifolds and their distance to the Earth, without any additional maneuvers, assuming that the same procedure can be applied with a very small amount of additional propellant, and therefore without changing substantially the cost of the transfer.

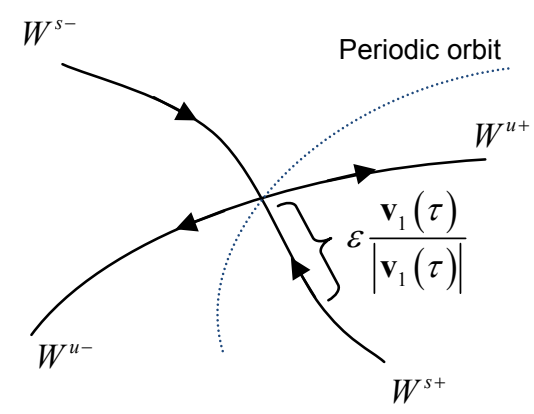

Fig. 19 Stable manifolds $\left(W^{\diamond}\right)$ wind onto the periodic orbit when integrated forward in time. On the contrary, unstable manifolds $\left(W^{u}\right)$ diverge from periodic orbit when integrated forward. These properties are reversed if the integration is performed backward in time. From (Koon et al. 2006)

In this paper we also assume that the sail, when present, is deployed once the spacecraft reaches the periodic orbit. Therefore, initial conditions of the manifolds are computed on the periodic orbit by using the full equations of motion, including the sail acceleration. However, the propagation of the manifold is then performed with $\mathbf{a}=0$ : thus, it is worth underlining that the manifolds that are found do not follow the same dynamics as the periodic orbit, and therefore strictly speaking they are not the manifolds associated to that orbit, except for the natural orbit family.

We compute and integrate backwards manifolds for all the orbits presented earlier, taking a number of points along each orbit. The integration time was set to one year, to limit the transfer time.

The following figures represent, for each orbit, the closest distance to the Earth that it is possible to achieve by following one of its manifolds. This value is plotted on the $x$ axis (in logarithmic scale) versus $z_{0}$ of each orbit (on the $y$ axis). The dashed black line at $185 \mathrm{~km}$ above the Earth represents the altitude of a hypothetical low Earth orbit, in which the spacecraft could be injected after launch. If one of the manifolds crosses this altitude, then a single upper stage is enough to inject the spacecraft onto the manifold. The spacecraft will then 
naturally move towards the target orbit. Once the insertion point is reached, the sail can be deployed and the periodic eight-shaped orbit achieved.

Fig. 20a shows the families with constant $\beta$. For all the families (other than $\beta=0.05$, whose shape makes it unsuitable for Earth observation), manifold transfer is possible to some orbits with $z_{0}$ in the range $(0.004,0.01)$. When $z_{0}>0.01$, no manifold passes in the vicinity of the Earth.

For the three families with constant period, see Fig. 20b, manifold transfers are possible for some orbits in the range $z_{0} \in(0.006,0.011)$, or when the $z$ amplitude is very limited, but these orbits are not interesting for polar observation.

The behavior of the manifolds of the orbits generated by tilting the solar sail is more disordered. As can be seen in Fig. 20c, small variations in the orbit may generate manifolds that collide with the Earth, or pass far away from it. 


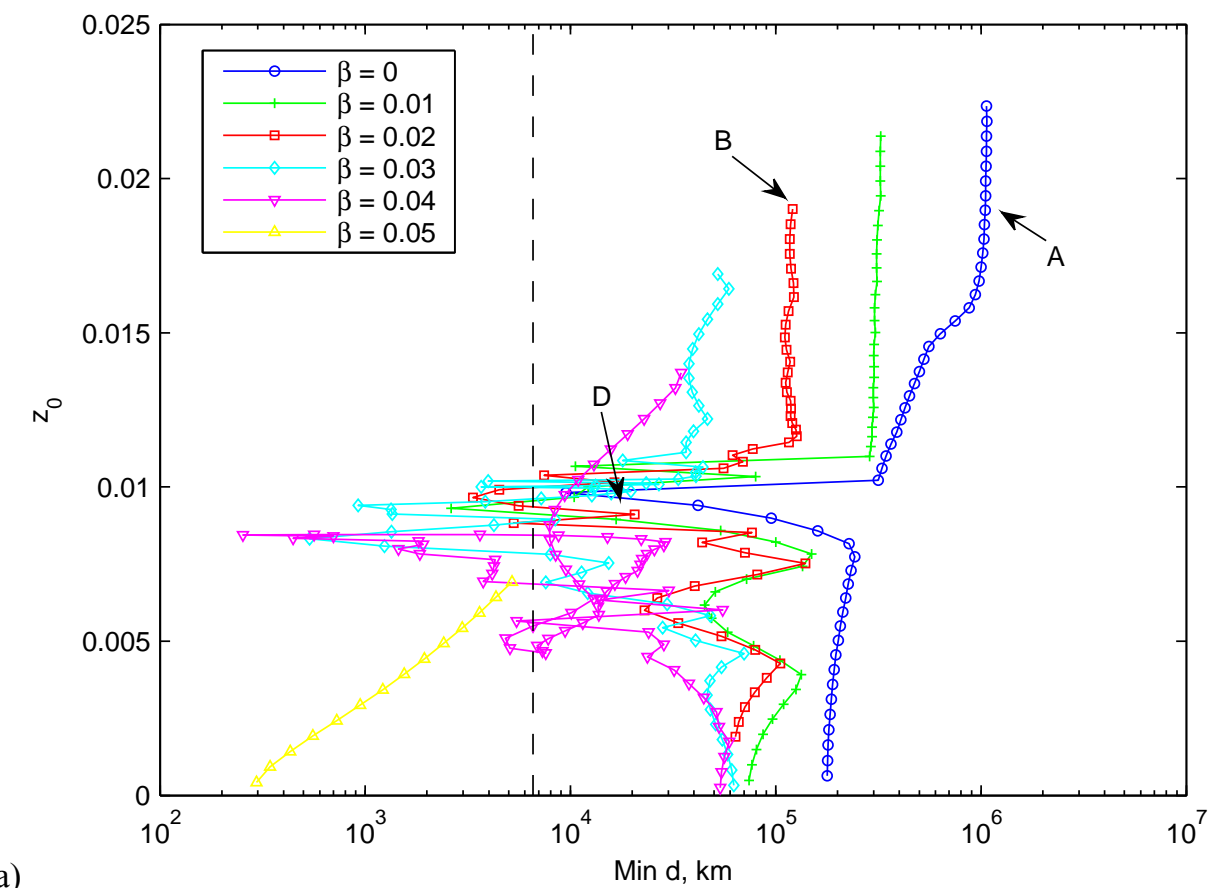

a)

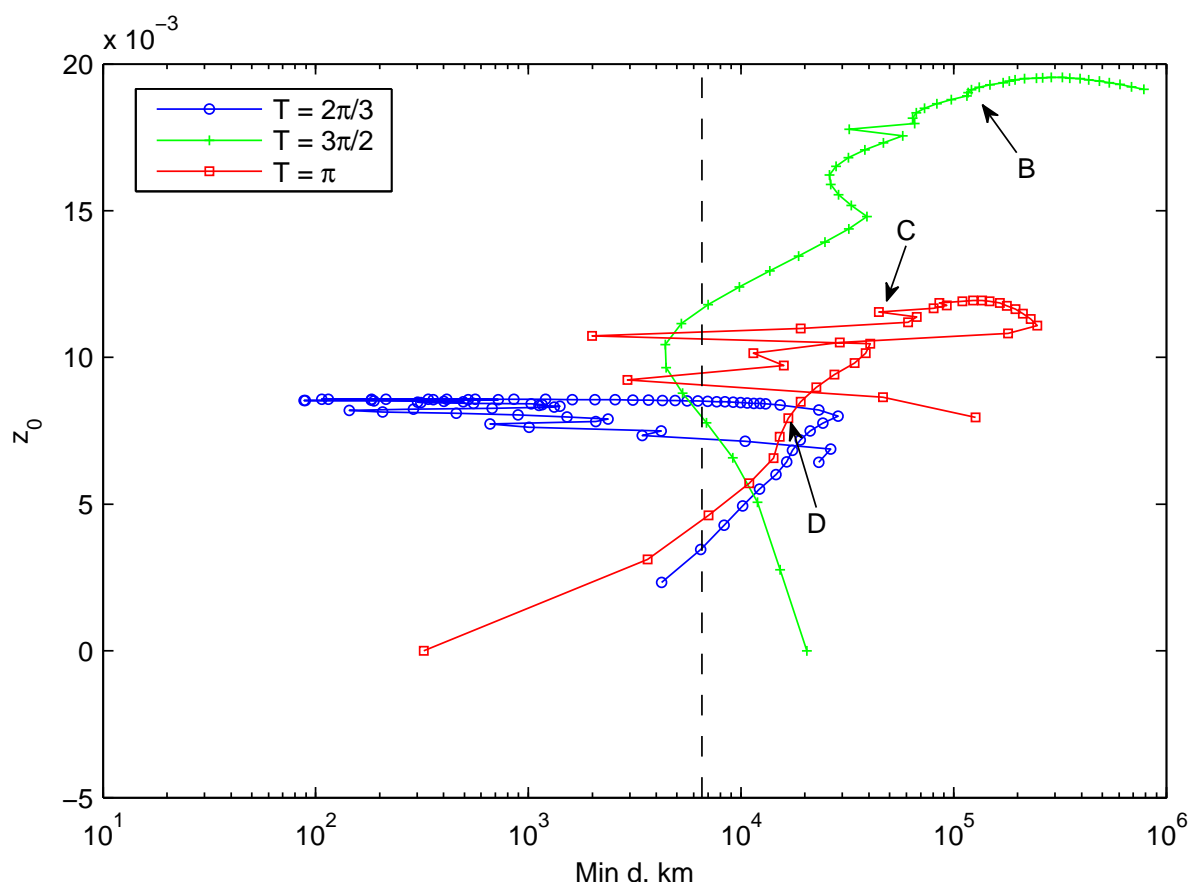

b)

Min d, km 


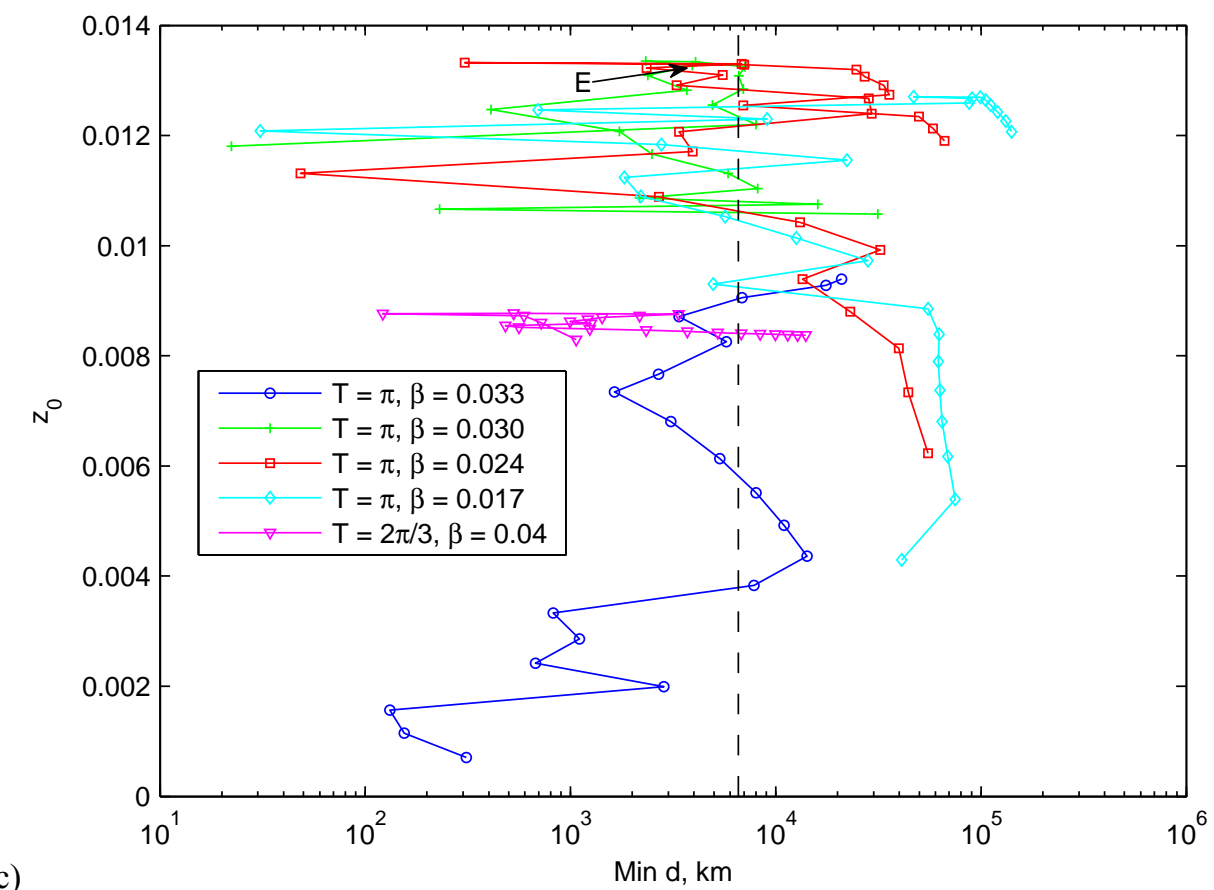

Fig. 20 Minimum distance to the Earth that a manifold can reach, departing from any point on the orbit, plotted versus the $z$ amplitude of the orbit. (a) Families of orbits with constant $\beta$; (b) Families of orbits with constant $T$; (c) Families of orbits with constant $T$ and $\beta$, varying the sail tilting

Considering the five orbits selected before, it is possible to conclude from the plots that manifold transfers to orbit $\mathrm{A}$ and $\mathrm{B}$ would certainly require a maneuver at the insertion point to be used, or a different transfer strategy has to be designed. Orbit $\mathrm{C}$ and orbit $\mathrm{D}$ do not have crossing points with LEO, however their manifolds pass very close to it, and therefore it is expected that a small maneuver (of the order of meters per second) at insertion point is sufficient to reach the LEO (Howell et al. 1994). Instead, natural transfers to orbit $E$ are feasible, selecting the appropriate manifold on that orbit.

Some plots of manifolds are provided here, to illustrate the type of transfers that they would enable. The three plots in Fig. 21 show the manifolds that are generated by three orbits in the natural family $(\beta=0)$. It is possible to see that for a particular amplitude of the orbit $z_{0}$, i.e. around 0.01 , the manifolds pass in the vicinity of the Earth. This corresponds to the minimum distance plotted in Fig. 20. Fig. 22 shows a trajectory that constitutes a possible transfer to orbit E. The insertion point on the orbit is highlighted with a star. 


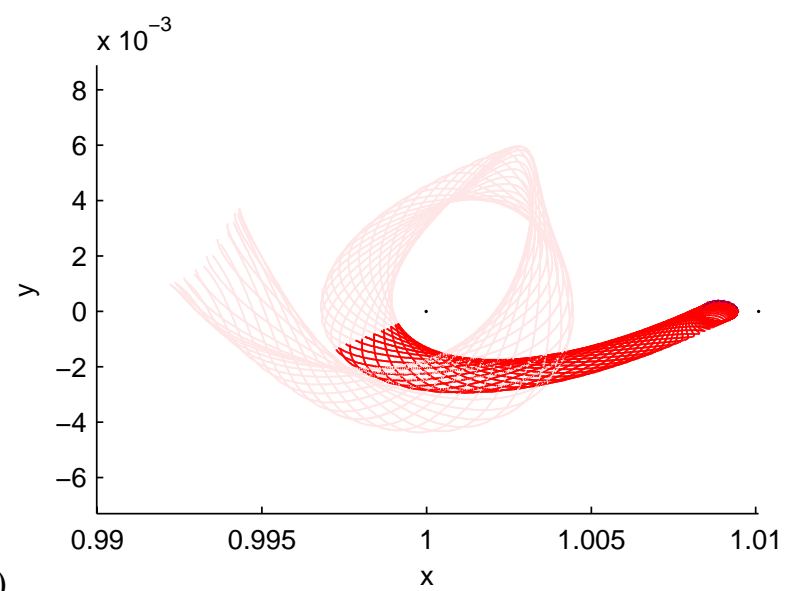

a)

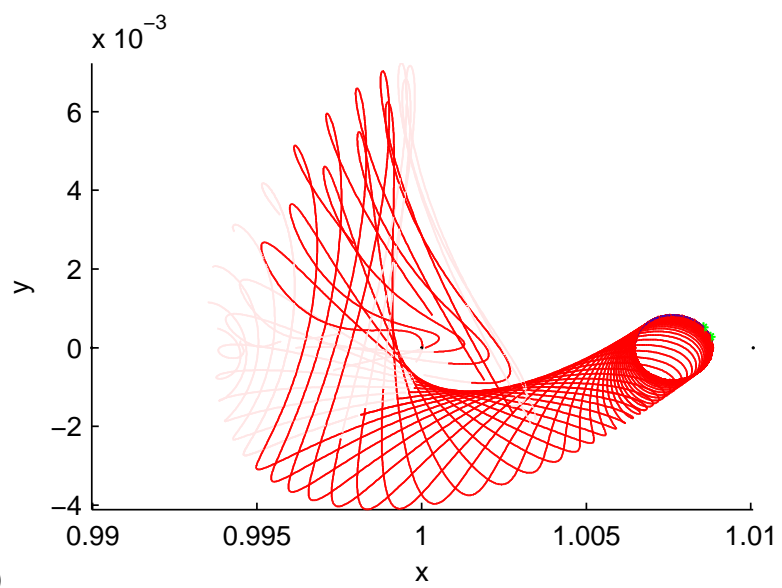

b)

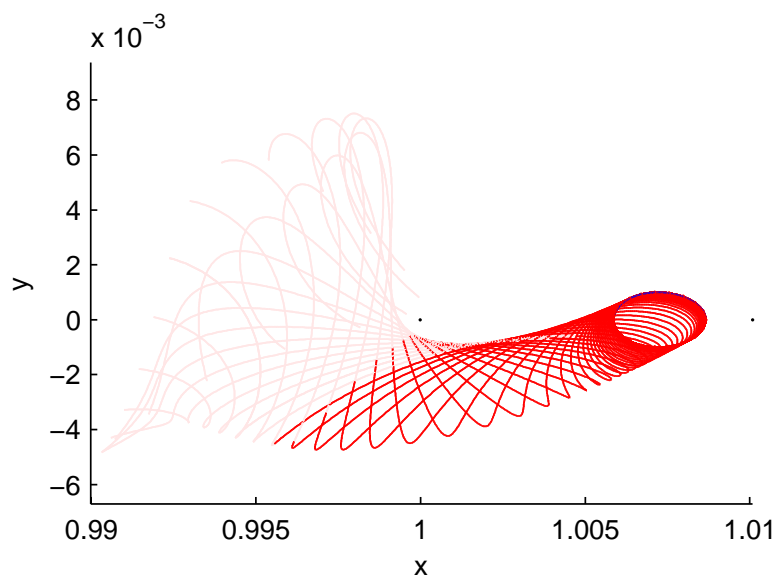

Fig. 21 Manifolds of three eight-shaped orbits of the natural family, of different $z$ amplitude: (a) $z_{0}=0.0069 ;$ (b) $z_{0}=0.0098 ;$ (c) $z_{0}=0.0106$. Manifolds are in solid color up to the closest point to the Earth within one year 


$$
\times 10^{-3}
$$

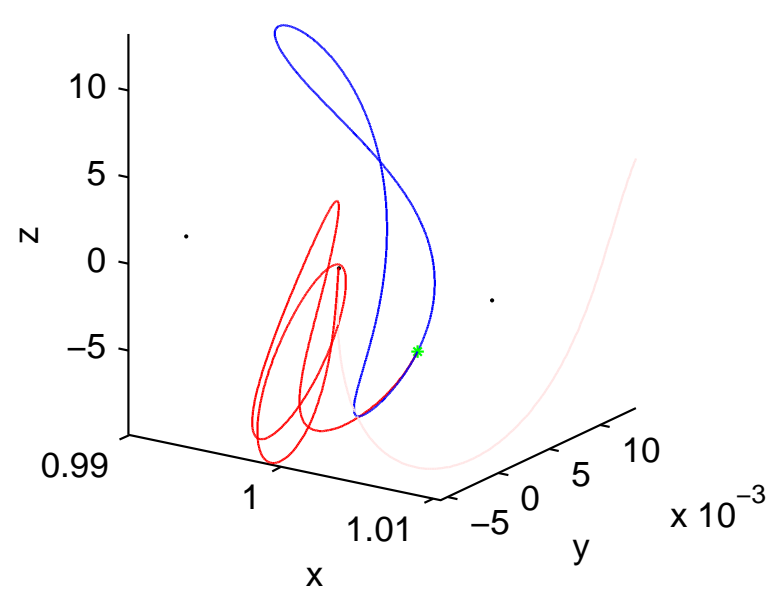

Fig. 22 A possible manifold transfer to orbit $E$. Transfer time is 245 days

\subsection{Orbit control through unstable manifolds}

The linearised dynamics around the orbits, and the study of the stable/unstable manifolds, can be the basis of alternative control strategies to the LQR. By investigating sail-displaced halo orbits, Farrés,Jorba (2012) proposed the method summarized here: the sail is kept at the reference orientation until the spacecraft, by following the natural evolution of the unstable manifold, will start to diverge from the reference orbit. At this point, a different sail orientation is sought, and therefore the dynamics of the system is changed, such that the spacecraft will be on a slightly different orbit, whose unstable manifold winds onto the original reference orbit. At this point, once again, the sail orientation is kept fixed. The spacecraft will eventually reach the reference orbit, at which point the sail is tilted again to the original orientation, and the process is repeated. This method was shown to be successfully applicable to a range of sail-displaced halo orbits, even when the full non-linear dynamics is used to propagate the spacecraft motion. Note that the structure of the monodromy matrix for the eight-shaped orbits is similar to that of the halo orbits, in particular involving two real eigenvalues associated with the stable/unstable manifold directions, as shown before. Due to this similarity, it is expected that the control technique proposed by Farrés,Jorba (2012) would be applicable for eight-shaped orbits. Current work is focusing on applying this type of control, and results on the controllability using this strategy will be the subject of a following publication. 


\section{Conclusion}

We have presented an overview of families of eight-shaped orbits that could be used for continuous Earth polar coverage. The orbits are either natural or displaced employing a sail of relatively modest lightness number $(<0.05)$. Long mission lifetimes can be achieved, as no propellant is necessary, and therefore, they constitute a nearer-term alternative to proposed missions such as Statites and pole-sitters.

Continuation methods were implemented and described, in order to construct families of orbits with varying period, lightness number or sail attitude. Due to the oscillation above and below the ecliptic plane, at least three spacecraft are required for continuous observation of both poles, and two for one pole only. By selecting specific orbits, continuous visibility to latitudes down to $70^{\circ}$ at any time of the day and year is possible, while visibility at even lower latitudes is available in particular seasons (when the poles are illuminated, for example). Furthermore, it is possible to select the period such that the visibility conditions repeat every year.

All natural and displaced orbits are found to be unstable, and a linear quadratic regulator $(\mathrm{LQR})$ was developed to show that is possible to control a spacecraft with modest effort. The instability was also exploited to design stable manifolds, which could represent an option for transferring from a low Earth orbit to the eight-shaped orbit with a single manifold injection maneuver. It was found that some of the orbits offer this possibility.

Future work will focus on two topics: the first is to investigate if it is possible to steer to solar sail actively along the orbit, in order to optimize the visibility conditions. The second is to design optimal sail transfers to those eight-shaped orbits in which the manifolds do not pass near the Earth.

\section{Acknowledgements}

This work was funded by the European Research Council, as part of project 227571

VISIONSPACE. The first author would like to thank Camilla Colombo and Joan Pau Sanchez for useful comments and discussions. 


\section{References}

Anderson, P.C., Macdonald, M.: Extension of Earth orbits using low-thrust propulsion. In: $61^{\text {st }}$ International Astronautical Congress (IAC 2010), Prague, Czech Republic, 27 Sept -1 Oct 2010 2010

Archambeau, G., Augros, P., Trélat, E.: Eight-shaped Lissajous orbits in the Earth-Moon system. MathS In Action 4(1), 1-23 (2011)

Baig, S., McInnes, C.R.: Artificial three-body equilibria for hybrid low-thrust propulsion. J Guid Control Dynam 31(6), 1644-1655 (2008). doi:10.2514/1.36125

Baig, S., McInnes, C.R.: Artificial halo orbits for low-thrust propulsion spacecraft. Celest Mech Dyn Astr 104(4), 321-335 (2009). doi:10.1007/s10569-009-9215-4

Baoyin, H., McInnes, C.R.: Solar sail orbits at artificial Sun-Earth libration points. J Guid Control Dynam 28(6), 1328-1331 (2005). doi:10.2514/1.14598

Broucke, R., Lass, H., Boggs, D.: A note on the solution of the variational equations of a class of dynamical systems. Celest Mech Dyn Astr 14(3), 383-392 (1976). doi:10.1007/bf01228524

Bryson, A.E., Ho, Y.-C.: Applied optimal control: optimization, estimation, and control (Revised printing). Taylor \& Francis Group, New York (1975)

Ceriotti, M., McInnes, C.R.: Hybrid solar sail and SEP propulsion for novel Earth observation missions. In: $61^{\text {st }}$ International Astronautical Congress (IAC 2010), Prague, Czech Republic 2010. International Astronautical Federation

Ceriotti, M., McInnes, C.R.: Generation of optimal trajectories for Earth hybrid pole-sitters. J Guid Control Dynam 34(3), 847-859 (2011). doi:10.2514/1.50935

Ceriotti, M., McInnes, C.R., Diedrich, B.L.: The pole-sitter mission concept: an overview of recent developments and possible future applications. In: $62^{\text {nd }}$ International Astronautical Congress (IAC2011), Cape Town, South Africa 2011

Dachwald, B., Mengali, G., Quarta, A.A., Macdonald, M.: Parametric model and optimal control of solar sails with optical degradation. J Guid Control Dynam 29(5), 1170-1178 (2006).

doi:10.2514/1.20313

Dichmann, D.J., Doedel, E.J., Paffenroth, R.C.: The computation of periodic solutions of the 3body problem using the numerical continuation software AUTO. In: International Conference on Libration Point Orbits and Applications, Aiguablava, Spain, 10-14 June 20022002

Driver, J.M.: Analysis of an arctic polesitter. Journal of Spacecraft and Rockets 17(3), 263-269 (1980). doi:10.2514/3.57736

Farrés, A., Jorba, À.: Periodic and quasi-periodic motions of a solar sail close to SL1 in the EarthSun system. Celest Mech Dyn Astr 107(1), 233-253 (2010). doi:10.1007/s10569-010-9268-4

Farrés, A., Jorba, À.: Station keeping of a solar sail around a halo orbit. In: 1st IAA Conference on Dynamics and Control of Space Systems (DyCoSS 2012), Porto, Portugal 2012

Folta, D., Young, C., Adam, R.: Unique non-Keplerian orbit vantage locations for Sun-Earth connection and Earth science vision roadmaps. In: Flight Mechanics Symposium, 1 June 2001 2001, pp. 41-51 
Forward, R.L.: Statite: a spacecraft that does not orbit. Journal of Spacecraft and Rockets 28(5), 606-611 (1991). doi:10.2514/3.26287

Funase, R., Mori, O., Tsuda, Y., Shirasawa, Y., Saiki, T., Mimasu, Y., Kawaguchi, J.: Attitude control of IKAROS solar sail spacecraft and its flight results. In: $61^{\text {st }}$ International Astronautical Congress (IAC 2010), Prague, Czech Republic 2010. IAF

Gómez, G., Jorba, A., Masdemont, J.J., Simó, C.: Study of the transfer from the Earth to a halo orbit around the equilibrium point $\mathrm{L}_{1}$. Celest Mech Dyn Astr 56(4), 541-562 (1993). doi:10.1007/bf00696185

Goudas, C.L.: Three-dimensional periodic orbits and their stability. Icarus 2, 1-18 (1963). doi:10.1016/0019-1035(63)90003-4

Hénon, M.: Vertical Stability of Periodic Orbits in the Restricted Problem. II. Hill's Case. Astronomy \& Astrophysics 30, 317-321 (1974)

Howell, K.C.: Families of orbits in the vicinity of the collinear libration points. J Astronaut Sci 49(1), 107-125 (2001)

Howell, K.C., Mains, D.L., Barden, B.T.: Transfer trajectories from Earth parking orbits to SunEarth halo orbits. In: Proceedings of the AAS/AIAA Spaceflight Mechanics Meeting. Part 1 (of 2), Febrary 14, 1994 - Febrary 16, 1994, Cocoa Beach, FL, USA 1994. Advances in the Astronautical Sciences, pp. 399-410. Publ by Univelt Inc

Kazantzis, P.G.: Families of three-dimensional axisymmetric periodic orbits in the restricted threebody problem: Sun-Jupiter case. Astrophysics and Space Science 61, 477-486 (1979a). doi:10.1007/BF00640543

Kazantzis, P.G.: Numerical determination of families of three-dimensional double-symmetric periodic orbits in the restricted three-body problem. I. Sun-Jupiter case. Astrophysics and Space Science 65(2), 493-513 (1979b). doi:10.1007/BF00648513

Kazantzis, P.G.: Numerical determination of families of three-dimensional double-symmetric periodic orbits in the restricted three-body problem. II. Sun-Jupiter case. Astrophysics and Space Science 69(2), 353-368 (1980). doi:10.1007/BF00661923

Koon, W.S., Lo, M.W., Marsden, J.E., Ross, S.D.: Dynamical systems, the three-body problem, and space mission design. Marsden Books, (2006)

Lappas, V.J., Wokes, S., Leipold, M., Falkner, P.: Attitude control design for solar sail missions. In: 6th International ESA Conference on Guidance, Navigation and Control Systems, Loutraki, Greece, October 17, 2005 - October 20, 2005 2005. European Space Agency, (Special Publication) ESA SP, pp. 191-194. European Space Agency

Lazzara, M.A., Coletti, A., Diedrich, B.L.: The possibilities of polar meteorology, environmental remote sensing, communications and space weather applications from Artificial Lagrange Orbit. Advances in Space Research 48(11) (2011). doi:10.1016/j.asr.2011.04.026 McInnes, C.R.: Solar sailing: technology, dynamics and mission applications. Springer-Praxis books in astronautical engineering. Springer-Verlag, Berlin (1999) McInnes, C.R., McDonald, A.J., Simmons, J.F.L., MacDonald, E.W.: Solar sail parking in restricted three-body systems. J Guid Control Dynam 17(2), 399-406 (1994) 
Moulton, F.R.: Periodic Orbits. vol. 161. Carnegie Institution of Washington Publications, Washington, USA (1920)

Senent, J., Ocampo, C., Capella, A.: Low-thrust variable-specific-impulse transfers and guidance to unstable periodic orbits. J Guid Control Dynam 28(2), 280-290 (2005). doi:10.2514/1.6398

Thurman, R., Worfolk, P.A.: The geometry of halo orbits in the circular restricted three-body problem. In., vol. Techinical report GCG95. University of Minnesota, Minneapolis, MN, (1996) Waters, T.J., McInnes, C.R.: Periodic orbits above the ecliptic in the solar-sail restricted threebody problem. J Guid Control Dynam 30(3), 687-693 (2007). doi:10.2514/1.26232

Wertz, J.R., Larson, W.J. (eds.): Space mission analysis and design, third edition. Microcosm press/Kluwer Academic Publishers, El Segundo, California, USA (1999)

Wie, B.: Solar sail attitude control and dynamics, part 1. J Guid Control Dynam 27(4), 526-535 (2004). doi:10.2514/1.11134

Zagouras, C., Markellos, V.V.: Axisymmetric periodic orbits of the restricted problem in three dimensions. Astronomy \& Astrophysics 59, 79-89 (1977) 